\title{
Supramolecular Gels and Functional Materials Research in India
}

\author{
Kalathil K. Kartha, Rahul Dev Mukhopadhyay, and Ayyappanpillai Ajayaghosh*
}

\begin{abstract}
Supramolecular gels are a class of soft materials made up of small molecules held together through non-covalent interactions. They have reversible properties and a wide range of applications. Chromophorebased gels are of particular interest due to their inherent electronic properties such as emission and charge transport useful for organic electronic device fabrication. Significant contributions have been made by Indian researchers in this area, which are highlighted in this mini review.
\end{abstract}

Keywords: Hydrogels · Organogels · Nanostructures · Self-assembly · Supramolecular chemistry

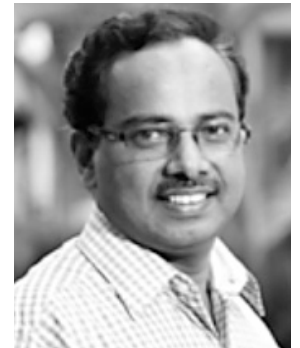

Ayyappanpillai Ajayaghosh is a CSIR-Outstanding scientist at the C S I R - N I I S T Trivandrum, and Dean and Professor of Chemical Sciences, Academy of Scientific and Innovative Research (AcSIR), New Delhi, India. He is a recipient of several awards including the Shanti Swarup Bhatnagar Prize 2007, Thomson Reuters Research Excellence-India Research Front Award 2009 and the Infosys Prize 2012. He is a fellow of all three science academies of India. His research interests include supramolecular and macromolecular chemistry, functional organic materials, functional dyes and fluorophores, photoresponsive materials, molecular assemblies and nanostructures, organogels, molecular probes for sensing and imaging etc. He serves as an associate editor of $P C C P$ and as an international advisory board member of Chemistry: An Asian Journal.

\footnotetext{
${ }^{*}$ Correspondence: Prof. A. Ajayaghosh Photosciences and Photonics Group

Chemical Sciences and Technology Division CSIR-National Institute for Interdisciplinary Science and Technology (CSIR-NIIST)

Trivandrum-695019, India

E-mail:ajayaghosh@niist.res.in
}

\section{Introduction}

Gel chemistry has been at center stage of soft materials research for the past three decades. Most of the early reports on the ability of certain molecules to gelate solvents came as the result of serendipity. Gel chemistry has its strong affiliation to supramolecular chemistry, and in particular to molecular self-assembly. Certain classes of molecules self-assemble in appropriate solvents to form soft non-flowing solid masses called gels. Over the years, a significant amount of research has gone into the in-depth understanding of the phenomenon of gelation. These studies have resulted in the development of a wide variety of molecules that are capable of gelating organic as well as aqueous solvents. Gels have a wide range of applications in food, cosmetics, catalysis, tissue engineering, sensing and in organic electronic devices. One-dimensional (1D) formation of fibrillar assemblies of molecules as a result of weak interactions such as H-bonding, $\pi$-stacking and van der Waals forces are responsible for the gelation of solvents. By the incorporation of chromophoric systems to gelator molecules, functional soft materials with electronic and photonic properties can be designed. Since gels are formed through weak interactions, gelation and associated properties are reversible. Such materials are expected to have applications as sensors for biologically and environmentally relevant analytes, security labels for documents and in bulk heterojunction solar cells. This mini review highlights the important developments in gel chemistry and functional materials research in India during the past three decades.

\section{Early Developments}

Indian chemists entered in the domain of gel chemistry during the late 1990s. At the Indian Institute Science (IISc.) Bangalore, Maitra and coworkers and Bhatacharya and coworkers independently started working with organogelators. A few years later Ajayaghosh and coworkers at CSIR - NIIST (formerly CSIR - RRL) started working in the area of organogelators based on $\pi$-systems. These studies have encouraged much research in the country to venture into the exciting area of gel chemistry and functional materials. Currently, a large number of research groups at various institutions in India are actively engaged in molecular assemblies, gel chemistry and functional materials research. These studies have resulted in a large number of publications in leading chemistry and materials journals and have also resulted in a large number of patents. The following sections cover the important contributions of the early stages.

\subsection{Bile Acid-based Gelators}

Maitra and coworkers have reported a number of gelators based on the bile acid platform. ${ }^{[1]}$ For example, the donor-substituted bile acids derivatives (1-3) were able to gelate organic solvents in the presence of trinitrofluorenone (TNF) as the acceptor (Fig. 1a). ${ }^{[2]}$ Compounds without hydrogen bonding linker form gels only in the presence of TNF. However, compounds with hydrogen bonding linkers formed gels in a variety of solvents through intermolecular hydrogen bonding and $\pi-\pi$ stacking even in the absence of TNF. ${ }^{[3]}$ Bile acid derivatives show similar packing patterns in bulk solid, xerogel and the gel in its native state. A supramolecular helical aggregate constructed from the pyrene gelator 4 (Fig. 1a) appended with a chiral alkyl chain provides a good balance between solubility and crystallization. ${ }^{[4]}$ Aggregation behavior as well as the stimuli-responsive properties of bile acid derivatives depend on the 
nature of the side chain appendages. $[1 \mathrm{~b}, 1 \mathrm{f}, 5]$ The mechanism of a tripodal cholic acid derivative to form an aqueous gel has been probed using a dye having two forms which can differentially bind to the hydrophobic pockets with a significant color change upon sol to gel transition. [6] The dynamics of the bound fluorescent dyes in a constrained environment depends on the chemical nature of the dye as well as the local polarity of the binding sites. ${ }^{[1 c, 7]}$

\subsection{Amino Acid-derivative-based Gelators}

Bhattacharya and coworkers have reported a simple amino acid-based derivative ( $N$-lauroyl-L-alanine), which was able to gelate the oil phase selectively from an oil-water mixture. ${ }^{[8]}$ The amide H-bonding and the long alkyl chain help the molecules to form a self-assembled fibrous structure which immobilizes the solvents (Fig. 1b). The large-scale synthesis of the gelator would have helped containment of oil spills, however, the requirement of heating and cooling to form the gel prevents the application. This problem was clearly identified by the authors and pointed out as a major challenge.

\section{$2.3 \pi$-Gelators Based on \\ Oligo(p-phenylenevinylene)s (OPVs) and Oligo(p-phenyleneethynylene)s (OPEs)}

OPVs are fluorescent $\pi$-systems used for a variety of applications. Ajayaghosh and coworkers have reported the self-assembly and gelation of OPV1 in 2001. ${ }^{\text {[9] }}$ The hydroxymethyl groups and the $\mathrm{C}_{12}$ $\mathrm{C}_{14}$ side chains were found crucial for the gelation of OPV1 in various aliphatic hydrocarbon solvents. Incorporation of chiral handles in OPV1 resulted in the formation of a relatively weak gel comprising helical fibers (Fig. 2a). ${ }^{[10]}$ Helicity could also be induced in the achiral OPV1 gelator by co-assembling with the chiral OPV gelator. In this case, the exciton coupled CD signal of the co-assembly was found to be stronger than that of the chiral gelator alone indicating a 'sergeant-soldier' effect on the chirality amplification. Symmetrical and unsymmetrical functionalization of OPVs with cholesterol moieties (Fig. 2b) allows a controlled supramolecular organization which results in helical nanoscopic architectures that display remarkable differences in optical, chiroptical, and morphological properties. ${ }^{[11]} \mathrm{A}$ pseudo H-type aggregation of the symmetrical derivative resulted in a twisted helix whereas a pseudo J-type aggregation of the unsymmetrical derivative gave a coiled helix. ${ }^{[12]}$ Weak dipolar interactions are also capable of facilitating gelation as noted in the case of the ester functionalized OPVs. Boc-Dand Boc-L-alanine linked OPV gelators in

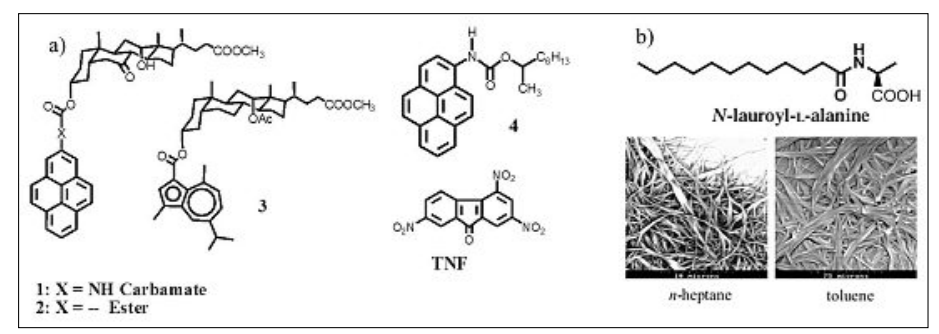

Fig. 1. (a) Chemical structures of bile acid derivatives and TNF. (b, top) N-lauroyl-Lalanine based gelator. (b, bottom) SEM images of N-lauroyl-Lalanine derived gel. Reproduced from ref. [8] by permission of the Royal Society of Chemistry @ 2001.

chloroform and toluene exhibited a morphology change from macroporous honeycomb to aligned fiber bundles. ${ }^{[13]}$ The origin of this morphology variation is attributed to the difference in the self-assembly pathways of these molecules in chloroform and toluene.

Interestingly, self-assembly of the oligo( $p$-phenyleneethynylene) derivative OPE1 (Fig. 2c) having the same kind of end functional group and alkyl side chains as that of OPV1, displayed vesicular assembly at lower concentrations and blue emitting gels at higher concentrations. ${ }^{[14]}$ Morphological study of the xerogels showed the formation of super bundled fibers. The chiral analogue (OPE2) of OPE1 did not form gels due to its high solubility and weak $\pi-\pi$ interaction. However, upon co-assembly of OPE2 with OPE1, gels were formed in nonpolar hydrocarbon solvents. The chirality of OPE2 was expressed in the co-assembly as supramolecular helicity, in the form of helical tubular assemblies (Fig. 2d). ${ }^{[15]}$

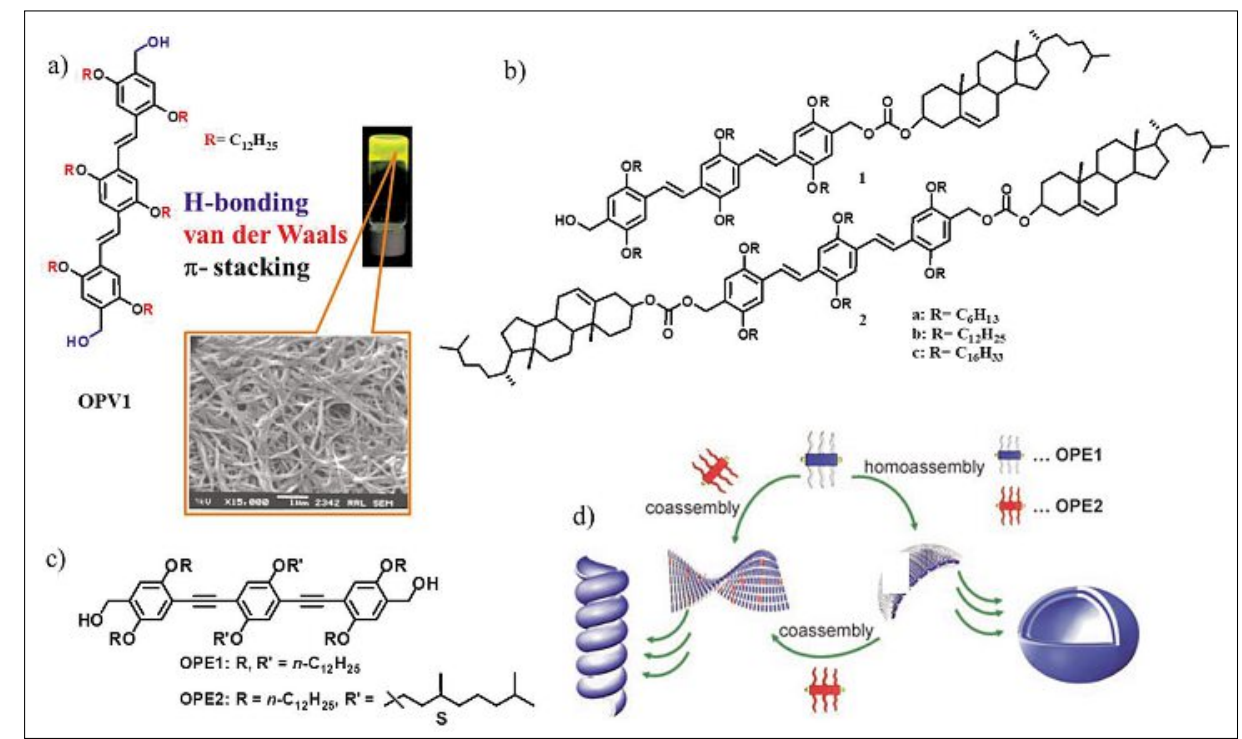

Fig. 2. (a) Chemical structure of OPV1, photograph and SEM image of the OPV1-decane gel. (b) Chemical structures of the cholesterol-based OPV gelators. (c) Chemical structures of the OPEbased gelators. (d) Schematic representation of homo- and co-assembly of the OPE gelators, OPE1 and OPE2. Reprinted with permission from ref. [15]. Copyright (2006) Wiley-VCH Verlag $\mathrm{GmbH} \&$ Co. KGaA, Weinheim.

\section{Later Developments}

The early success with the design of new gelator molecules and the progress made elsewhere in gel chemistry have prompted many research groups in India to develop organo- and hydrogelators of a variety of molecules. These include chromophore-based gelators, amino acid- and peptide-based gelators, organic salt-based gelators and hybrid gelators. Among these, linear $\pi$-system based gelators have attracted much attention due to their reversible optical and electronic properties. This section will cover important contributions during the period of 2005-2010.

\subsection{Gels Based on Complementary H-Bonding}

Multiple H-bonding moieties such as melamines and cyanurates help molecules self-assemble through strong complementary interactions providing different architectures. ${ }^{[16]}$ Incorporation of chromophoric moieties with complementary synthons facilitates linear and circular organization of chromophores. For example, the melamine-linked OPE derivative (M-OPE) (Fig. 3a) and the cyanurate (CU) (Fig. 3a) 
co-assemble to form a rosette assembly at lower concentration, which further selfassemble to form donut-shaped circular objects. ${ }^{[17]}$ At higher concentration they were able to gelate organic solvents. An OPV derivative of a barbituric acid (BAR) with a hydrogen-bonding headgroup, and a wedge-shaped tridodecyloxybenzyl (TDB) tail, self-assemble to form closed ringshaped nanostructures (nanorings) with uniform dimensions. ${ }^{[18]}$ The OPV-dimer with multiple $\mathrm{H}$-bonding interaction sites exhibits a guest-induced transformation of well-defined nanostructures. A chiroptical input into the nanoassembly could be possible by incorporating chiral guest molecules. ${ }^{[19]}$ The role of a cyanurate in the selfassembly of a melamine-linked TPE was studied thereby modifying the physical properties and morphological features of the resultant supramolecular gels. ${ }^{[20]}$ The self-assembly of TPE in the absence and presence of a complementary cyanurate (dCA) was demonstrated, in which a 1:1 complex TPE:dCA, formed a transparent gel (Fig. 3b). On the other hand, hierarchical self-assembly of TPE under higher concentrations resulted in blue emitting gels in aliphatic solvents such as hexane and methylcyclohexane (MCH).

Nandi et al. have extensively investigated a series of two-component systems such as melamine-riboflavine, melaminegallic acid and melamine-lumichrome which form thermo-reversible hydrogels through complementary hydrogen bonding. [21] The melamine-riboflavin system (Fig. 3c) showed enhanced luminescent properties in the gel state, particularly at 1:3 molar ratio. The gelation mechanism has been explained using optical, electron and atomic force microscopy together with circular dichroism and photoluminescence spectroscopy.[21a,21b] By changing the composition of the gelator molecules, macro morphology of the gels could be changed from helical fibers to rods and finally to hollow tubes. The hollow tubes exhibited a higher PL intensity than that of the helical fibers, thus modulating the 1D macro-morphology and the luminescent properties of the assemblies.

Nandi et al. have also reported a thermo-reversible gel of melamine-coordinated silver(I) in water with low minimum gelation concentration of $0.6 \% \mathrm{w} / \mathrm{v}$ at $30^{\circ} \mathrm{C}$. Mixing silver nitrate with melamine at high temperature in water followed by cooling resulted in the formation of hydrogels having 1:1 and 1:2 molar compositions of silver and melamine (AgM11 and AgM12). Interestingly, the 1:2 compositions of $\mathrm{Ag}$ and melamine formed a stable gel, but the 1:1 composition formed a metastable gel that crystallizes upon aging. The gelation ability of AgM12 was highly dependent on counter anions of $\operatorname{Ag}(\mathrm{I})$, and the hydrogel
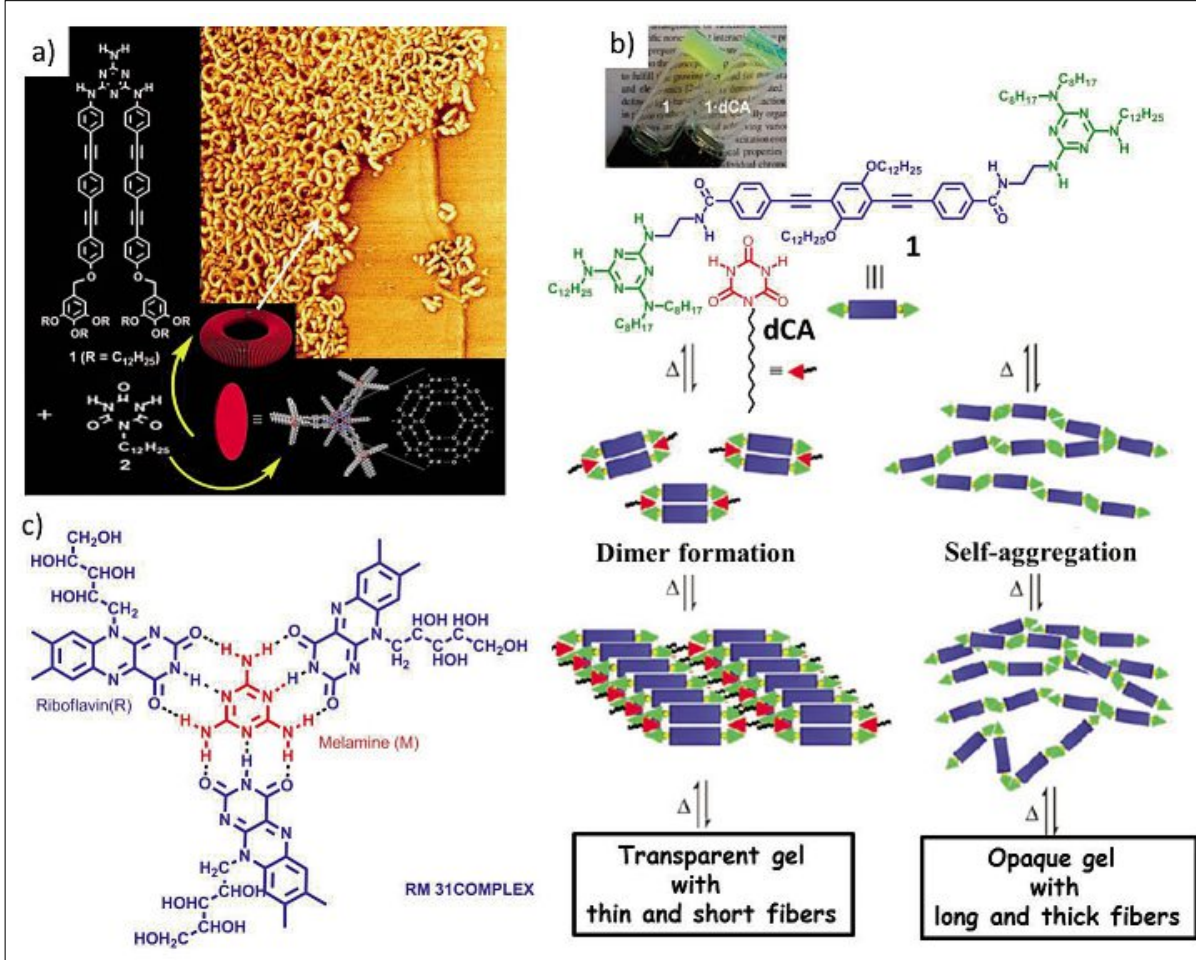

Fig. 3. (a) Schematic representation of rosette assembly formations from the melamine-linked OPE derivative and cyanurate (inset: AFM image of donuts). Reprinted with permission from ref. [17]. Copyright (2008) Wiley-VCH Verlag GmbH \& Co. KGaA, Weinheim. (b) Gel photographs and schematic representation of TPE and TPE:dCA self-assembly. Reproduced from ref. [20] with permission of the Royal Society of Chemistry (C) 2009. (c) Schematic representation of a melamineriboflavine complex.

was stable at neutral $\mathrm{pH}$. The sol-gel transition of AgM12 hydrogel could be tuned by the addition of equivalent amounts of halogen anions and thereby the gel is disrupted to a sol with the precipitation of $\mathrm{AgX}$. Further addition of one equivalent $\operatorname{Ag}(\mathrm{I})$ followed by heating the solution led to a homogeneous solution. On subsequent cooling the gel reappeared.[22]

\subsection{Organic Salt-based Gels}

Understanding the molecular interactions present in a crystal helps in the identification of supramolecular synthons, in which the special interactions are robust enough to predict the structure of the resulting crystalline solid. Dastidar and coworkers have significantly contributed to the development of organic salt-based gelators. [23] Attempts at crystallizing the salt $\mathbf{G 3 3}$ from

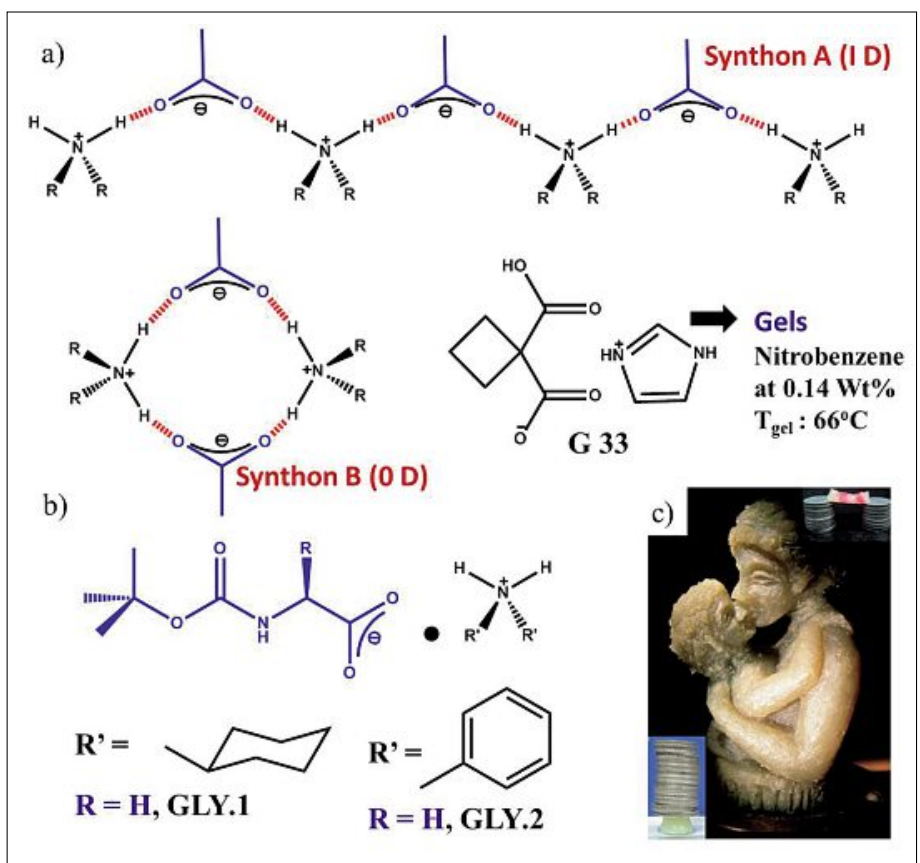

Fig. 4. (a, b)

Secondary ammonium monocarboxylate (SAM) synthon. (c) A gel sculpture of 'mother and child' made from a $7.0 \mathrm{wt} \%$ nitrobenzene gel of GLY.1. (inset: top right - self-healing of five gel blocks of GLY.1, bottom left - the freestanding gel of GLY.1 withstanding the pressure of 13 Indian 5 Rupee coins $\sim 117$ g). Reprinted with permission from ref. [26]. Copyright (2012) Wiley-VCH Verlag $\mathrm{GmbH} \& \mathrm{Co}$. KGaA, Weinheim. 
nitrobenzene resulted in a gel.[24] This serendipitous observation led to a number of reports from the same group exploring the secondary ammonium monocarboxylate (SAM) synthon, which displays either 1D (synthon A) or 0D (synthon B) like supramolecular arrangement (Fig. 4a). ${ }^{[25]}$ Recently, the same group reported a library of simple organic salts derived from t-butoxycarbonyl (Boc)-protected L-amino acids and two secondary amines (dicyclohexyl- and dibenzyl amine). A nitrobenzene gel of one of the salts of dicyclohexyl amine (GLY.1) revealed remarkable load-bearing, moldable and self-healing properties, where its dibenzyl ammonium counterpart (GLY.2) failed to display such properties (Fig. 4b, c). [26]

Secondary ammonium dicarboxylate (SAD) salts give rise to $1 \mathrm{D}$ hydrogen bonding networks (HBNs), where propagation of synthon $\mathrm{B}$ in one dimension is possible, virtually due to the presence of two $\mathrm{COO}^{-}$moieties (Fig. 5a). The same concept was put to use in gaining access to low molecular weight organometallic gelators, derived from a salt of ferrocene-1,1'-dicarboxylic acid (FDCA) and dicyclohexyl amine (DCHA). Scanning electron microscopic investigation of the xerogel revealed an entangled fibrillar network consisting of twisted fibers of both handedness. ${ }^{[27]} \mathrm{A}$ clever exploitation of the SAD synthon gave access to a few chiral gels derived from secondary ammonium salts of $(1 R, 3 S)-(+)$-camphoric acid. ${ }^{[28]}$

The primary ammonium monocarboxylate (PAM) synthon forms $1 \mathrm{D} \mathrm{HBNs}$ in most cases and a 2D hydrogen-bonded sheetlike structure in rare cases. Soundinduced instant gelation of petrol and other solvents was observed for one of the PAM salts. ${ }^{[29]}$ Various dicarboxylate salts derived from cyclobutane-1,1-dicarboxylic acids and primary alkyl amines $\left(\mathrm{CH}_{3}-\right.$ $\left.\left(\mathrm{CH}_{2}\right)_{\mathrm{n}}{ }^{-} \mathrm{NH}_{3}, \mathrm{n}=3-15\right)$ were further studied and it was seen that salts with $\mathrm{n}>10$ were excellent gelators for petrol, diesel and kerosene. ${ }^{[30]}$ The primary ammonium dicarboxylate (PAD) synthon has also been used to generate a few ferrocene dicarboxylic acid-based metallogels. [31] The PAD synthon was further explored to obtain a new series of gelators which revealed reverse-thermal gelation behavior. ${ }^{[32]}$ A combinatorial library approach for the screening and identification of potential organic salt-based gelators was adopted making use of the $\mathrm{SAD}^{[33]} \mathrm{PAM}^{[34]}$ as well as the PAD synthons. ${ }^{[35]}$ A new series of diprimary ammonium monocarboxyate (DPAM) salts was reported to form gels in various solvents.[36]

\subsection{Peptide-based Gelators}

Amino acids and peptides are a good platform for the design of molecular

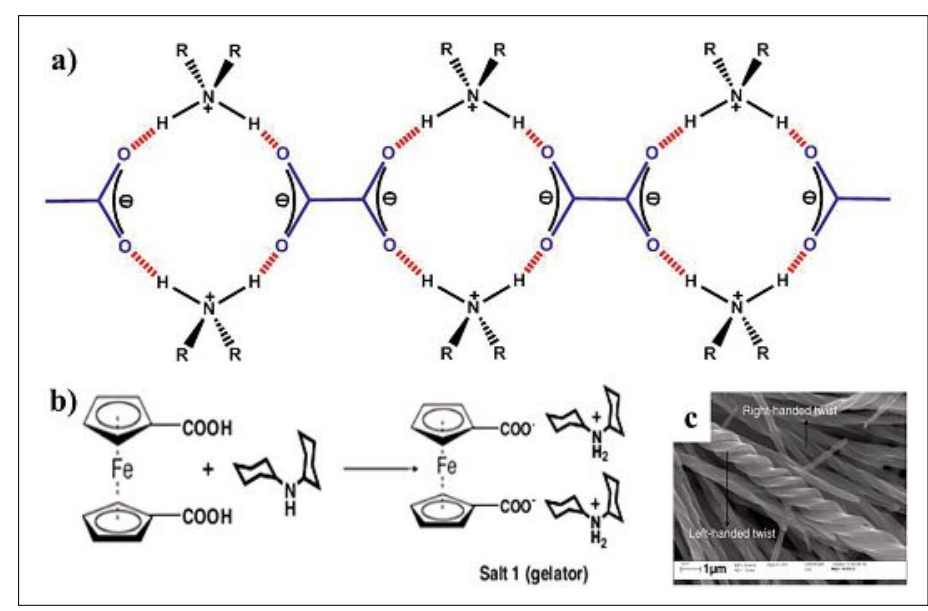

Fig. 5. (a) Secondary ammonium dicarboxylate (SAD) synthon. b) Organometallic low molecular weight gelator. (c) SEM micrograph of the DMF-xerogel of $1: 1$ salt of FDCA and DCHA. Reprinted from ref. [27]. Copyright (2008) with permission from Elsevier.

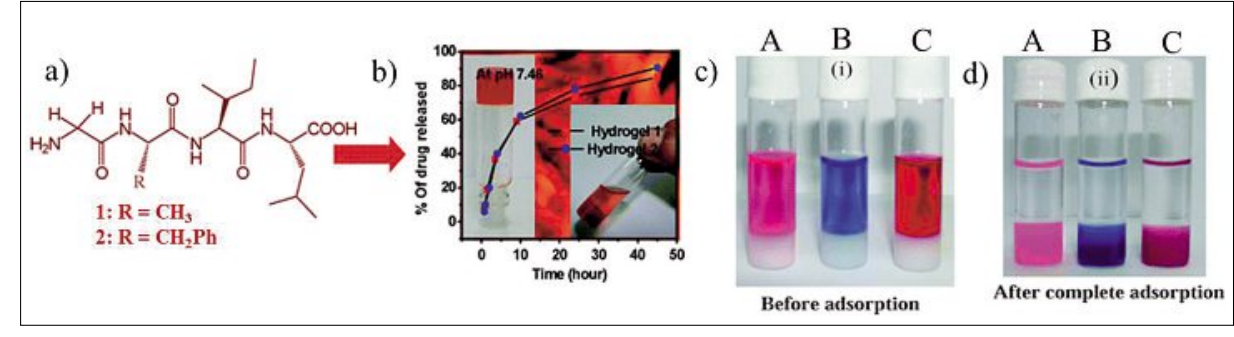

Fig. 6. (a) Chemical structures of tetrapeptide-based hydrogelators (1 and 2), (b) Drug release profile of 1 and 2 at pH 7.46, photographs and AFM image (background) of hydrogel 1. Reprinted with permission from ref. [37]. Copyright (2009) American Chemical Society. Photograph of hydrogel obtained from gelator peptide: (c) before adsorption of dye molecules and (d) after complete adsorption of dye molecules (A: Rhodamine B, B: Reactive blue 4, C: Direct red 80). Reproduced from ref. [39] with permission of the Royal Society of Chemistry (C) 2009

gelators. Banerjee et al. have developed synthetic tetrapeptide $\left(\mathrm{NH}_{2}-\mathrm{Gly}-\mathrm{X}\right.$-IleLeu-COOH, $\mathrm{X}=$ Ala/Phe, Fig. 6) based supramolecular hydrogels at physiological $\mathrm{pH} .{ }^{[37]}$ Replacement of the Ala residue by aromatic as well as hydrophobic phenylalanine (Phe) residues has been found to enhance the gelation efficiency. These gelator molecules were assembled through hydrogen bonding, aromatic-aromatic interactions and other non-covalent interactions to form an anti-parallel $\beta$-sheet structure. The potent anticancer drug doxorubicin could be encapsulated within the hydrogel matrix for sustained release of the drug at physiological $\mathrm{pH}$. The proteolytic stability of a bioactive molecule-releasing peptide hydrogel could be significantly increased by the judicious incorporation of a non-protein, naturally occurring amino acid $(\beta$-alanine) in place of $\alpha$-alanine amino acid residue in a dipeptide-based hydrogelator. ${ }^{[38]}$ These proteolytically stable hydrogel materials have been used for the encapsulation and sustained release of water-soluble vitamins $\mathrm{B}_{2}$ and $\mathrm{B}_{12}$ at physiological $\mathrm{pH}$. Some of the tripeptide-based hydrogel materials have been found useful for the treatment of waste water. ${ }^{[39]}$

\subsection{Thiophene-derived Gelators}

Recently, the first example for thienylenevinylene-based gels that form aligned supramolecular wires on freshly cleaved mica surfaces through epitaxial assembly has been reported (Fig. 7). ${ }^{[40]}$ Microscopic analysis showed that the OTV1 gel was comprised of entangled micrometer-sized fibers. For more insight into the electronic properties of the self-assembled OTV1 aggregates, charge carriers were generated photolytically by adding $N, N^{\prime}$ bis(2,5-di-tert-butylphenyl)-3,4,9,10perylenedicarboximide (PDI) as an electron acceptor. Flash photolysis time-resolved microwave conductivity (FP-TRMC) and transient absorption spectral (TAS) studies proved the high charge carrier mobility in the self-assembled state when compared to the monomeric state, highlighting the importance of self-assembly and gelation on the electronic properties (Fig. 7c). This study highlights the role of hydrogen bonded and $\pi$-stacked molecular assemblies in improving the electronic properties of thiophene-based systems. An increase in the conjugation length of the OTV molecular wires has a remarkable influence on the gelation and the conducting properties. Electrical conductivity of these new p-type semiconduting gels could be improved to the metallic range by increasing the conjugation length and by doping with iodine (Fig. 7d). The absorption spectra exhibited significant changes in presence of iodine vapors which are predominant in the case of OTV2 and OTV3. The intensity of the polaron bands increases with the iodine ex- 


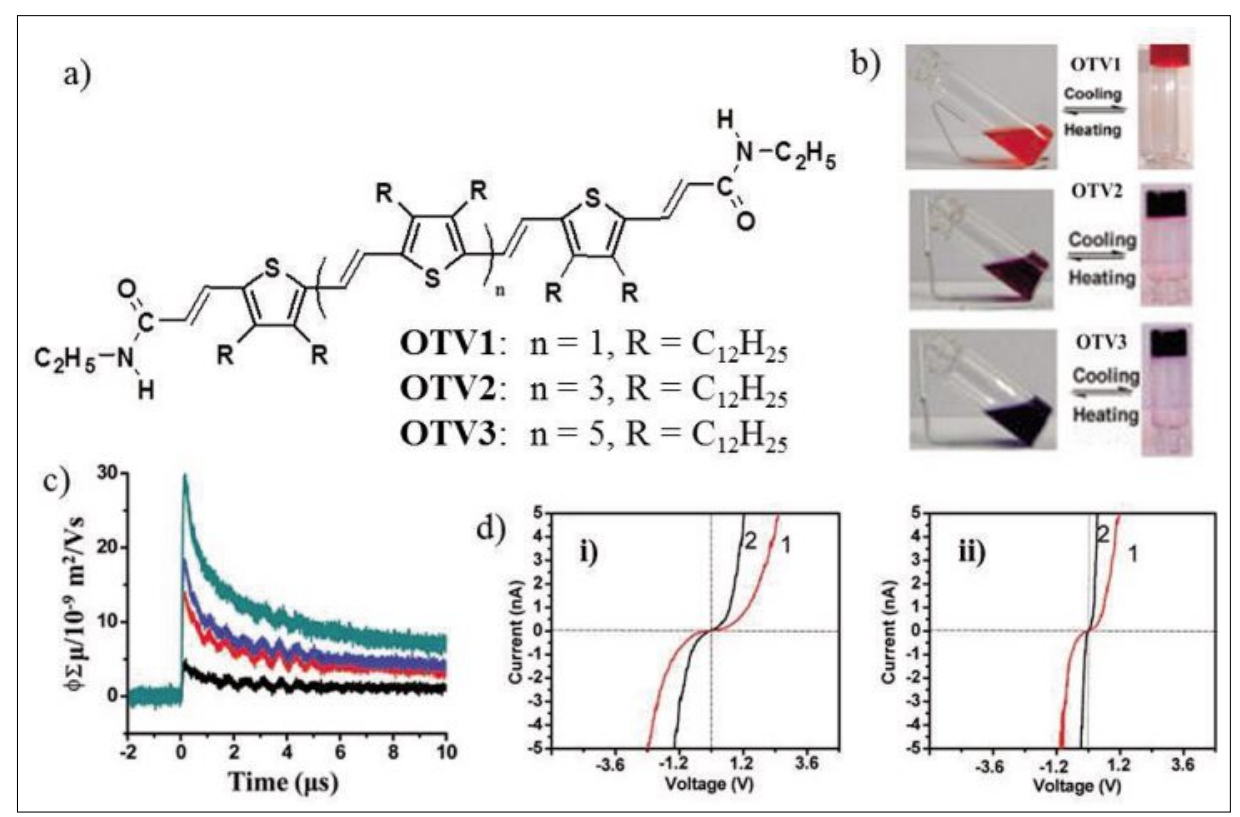

Fig. 7. Chemical structures (a) and photographs of sol-gel transition of OTV1-3 (b) (c) FP-TRMC transient conductivity profiles $\left(\lambda_{\mathrm{ex}}=355 \mathrm{~nm}\right)$ of OTV1 with different weight fraction of PDI prepared from $n$-decane/chloroform (1:1 v/v) solutions. (d) C-AFM measurements of OTV2 and OTV3 xerogels from decane solution drop cast on HOPG $\left(\mathrm{c}=1 \times 10^{-4} \mathrm{M}\right)$. I-V curves of undoped (i) and doped (ii) xerogels of OTV2 (i) and OTV3 (ii). Reprinted with permission from ref. [40] and ref. [41]. Copyright (2010) American Chemical Society.

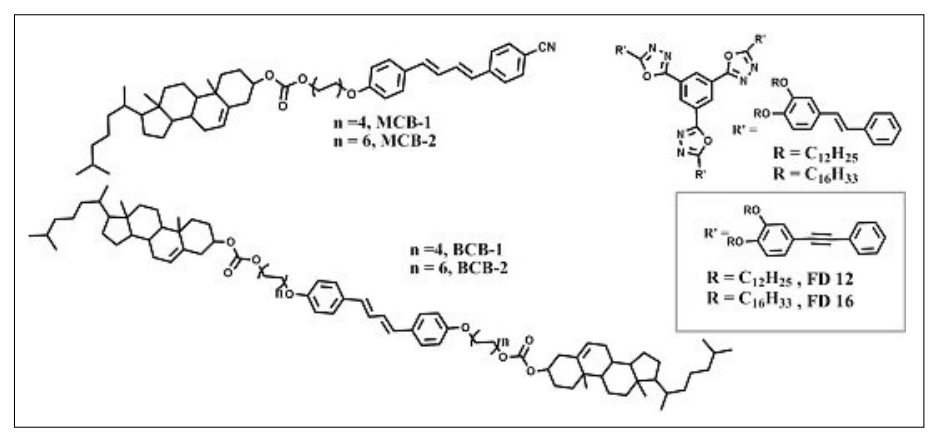

Fig. 8. Chemical structures of different butadiene and oxadiazole derivatives.

posure time and are saturated within $1 \mathrm{~h}$ of exposure. The bulk electrical conductivities of OTV1-3 xerogels on glass substrate before and after doping with iodine vapors were measured by four-probe dc resistivity method. The electrical conductivity of $4.8 \mathrm{~S} / \mathrm{cm}$ observed for the iodine-doped OTV3 xerogel is one of the highest values reported for a molecular gelator. ${ }^{[41]}$

\subsection{Liquid Crystal-based Gels}

The construction of supramolecular architectures by the spontaneous self-assembly of butadiene molecule was reported by Das et al. These derivatives formed transparent gels in polar solvents, such as alcohols, acetonitrile, and dimethyl sulfoxide (DMSO). The mechanism of gel formation involves a spontaneous hierarchical self-assembly of such derivatives, which leads to the formation of smaller vesicles at the initial stages, that merge to form larger ones until they finally link together to form gels consisting of globular aggregates with solvent entrapped within them. ${ }^{[42]}$ A striking difference in the nature of the self-assembly process between molecules consisting of diphenyl butadiene derivatives linked via flexible alkyl chains to one or two cholesterol units was also reported (Fig. 8). ${ }^{[43]}$ These differences may be attributed to the nature of the molecular aggregates formed that consisted of J-type aggregates in case of the mono-cholesterol derivatives and $\mathrm{H}$-type aggregates for the bis-cholesterol derivatives.

A novel class of octupolar oxadiazole derivatives has been synthesized and their mesophase properties and ability to selfassemble in nonpolar solvents were examined. Unlike conventional organogelators, disk-shaped molecules have to selforganize in a manner so as to maximize the $\pi-\pi$ interactions which can result in the formation of columnar superstructures leading to the formation of fibers and eventually entangled network of fibers wherein the solvent molecules are immobilized.[44] Star-shaped molecules consisting of a 1,3,4-oxadiazole core derivatized with alkoxy-substituted phenyl ethynylenes, FD12 (dodecyl) and FD16 (hexadecyl) were reported (Fig. 8). [45] The length of the alkyl substituent was observed to have a significant effect on the absorption and fluorescence properties of the gels, which was attributable to the role of the alkyl substituents in controlling the nature of the molecular packing within the self-assembled fibers of the gels.

\subsection{Hybrid Gelators}

One of the main drawbacks of supramolecular gels is their poor stability above ambient conditions. For example, many of the self-assembled gels are thixotropic in nature. One of the solutions to improve the stability of the gels is to reinforce them with suitable and compatible materials such as carbon nanotubes, nanoparticles or with covalent polymers. In this way hybrid materials having properties different from the individual components can be designed. $\pi$-Systems are ideal materials to prepare hybrid gelators.

\subsubsection{Carbon Nanotube-based Gels}

Ajayaghosh et al. have reported that the stability of OPV gels could be improved by the addition of single-walled or multiwalled carbon nanotubes. Interestingly, gelation of OPV occurred below the critical gelator concentration upon addition of the carbon nanotubes (CNT). ${ }^{[46]}$ CNTs are entrapped in the self-assembly thereby reinforcing the gel fibers (Fig. 9a). The self-assembly facilitates the creation of a hydrophobic coating of OPVs on CNTs with a surface topography consisting of micrometer-sized hills and valleys with a nanoscale coating of hairy hydrocarbon chains akin to lotus leaves. The fact that superhydrophobicity was exhibited by the relatively cheaper MWNTs, when compared to SWNTs, broadens the scope for potential applications of the composite. ${ }^{[47]}$

Bhattacharya et al. have studied the supramolecular interactions of organogelators with various carbon nanomaterials (CNMs), such as functionalized graphenes, single-walled carbon nanotube (SWNT) and fullerene $\left(\mathrm{C}_{60}\right) \cdot{ }^{[48]}$ Rheological and electrical conductivity studies revealed that the nanocomposite gels have improved properties compared to the native gels. This study revealed that the molecular level interaction between CNMs and fibrous network of gelator molecules is responsible for the improved properties.

\subsubsection{Metal Nanoparticle-based Gels}

Hybrid materials based on inorganic nanoparticles and $\pi$-conjugated oligomers have received significant attention due to their optoelectronic properties. Hybrid OPV1/OPV6-Au supramolecular tapes have been prepared by mixing OPV1 with OPV6-Au derivative in toluene followed by heating the mixture above the gelation temperature of OPV1 followed by cooling. ${ }^{[49]}$ The key feature of the hybrid self- 


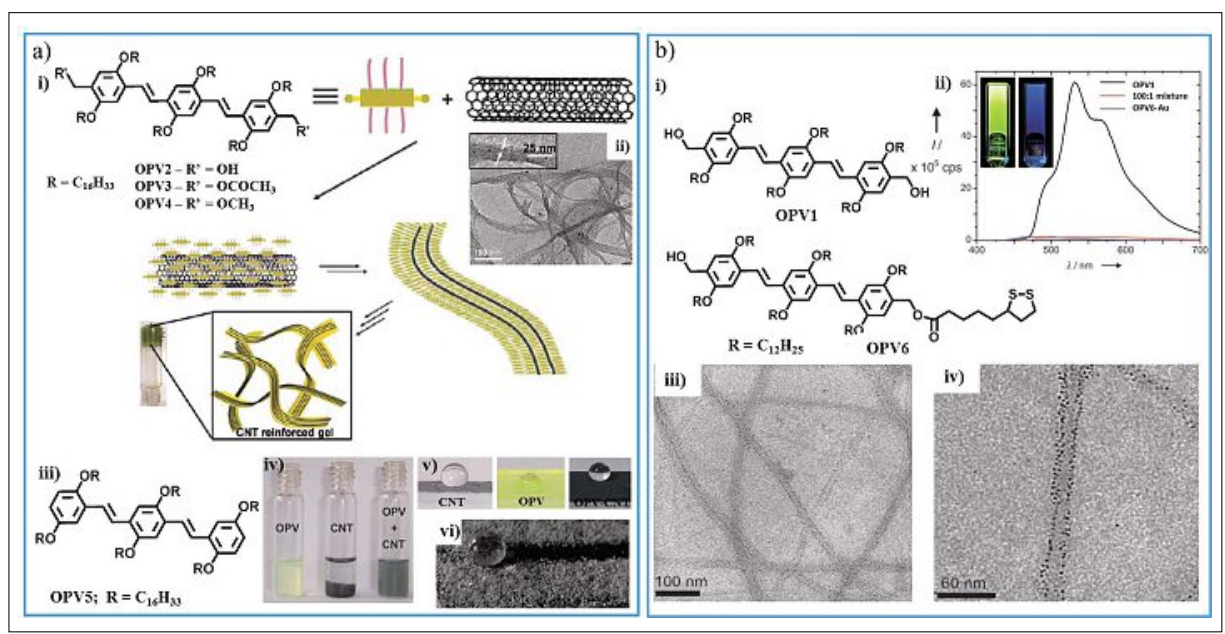

Fig. 9. (a) Chemical structures of OPVs (i and iii), HR-TEM image of OPV-CNT hybrid (ii) and a schematic representation of OPV-CNT gel formation. Reprinted with permission from ref. [46]. Copyright (2008) Wiley-VCH Verlag GmbH \& Co. KGaA, Weinheim. Photographs of OPV1, CNT and OPV-CNT solutions (iv), a water droplet on CNT, OPV1, and OPV1-CNT nanocomposite coatings (v). vi) Image showing the self-cleaning ability of a dusted composite surface. Reprinted with permission from ref. [47]. Copyright (2008) Wiley-VCH Verlag GmbH \& Co. KGaA, Weinheim. (b) Chemical structure OPVs (i). Fluorescence spectra of OPV1 gel and 100:1 OPV1 and OPV6-Au hybrid gel (inset: respective photographs) (ii). TEM images at different magnifications of the OPV1/ OPV6-Au (100:1) tapes deposited from toluene (iii and iv). Reprinted with permission from ref. [49]. Copyright (2007) Wiley-VCH Verlag GmbH \& Co. KGaA, Weinheim.

assembly was the functionalization of the nanoparticles with ligands which can interact with the OPV1 tapes through non-covalent interactions similar to those that hold the tape together (Fig. 9b). Pradeep et al. have reported the detailed transformation on the hybrid assembly of gold nanoparticles and OPV gelators. ${ }^{[50]}$

The interaction of low molecular weight organogelators (LMOGs), end capped with nanoparticles and gold nanoparticles (AuNP) capped with n-alkanethiols, cholesterol-based thiol and $p$-thiocresol has been studied. Hybrid gels comprising of capped AuNP and gelator (Fig. 10) in 1:1 (w/w) ratio exhibited improved viscoelastic behavior when compared to the native gel in toluene. The interdigitation of alkyl chains of capped AuNP with the gelator molecules was responsible for the enhanced mechanical strength of the hybrid gel. The salt-bridging interactions between carboxylates of various fatty acids and ammonium ions of a variety of amines have evolved as a medium to grow nanoparticles over the fibers of the two component gels. ${ }^{[51]}$ These gels possessed hydrophilic pockets over the gel fibers which led to the controlled growth of the nanoparticle assembly. Investigation of keto-enol tautomerism in gels was possible with an organogelator. [52] Further, self-assembly of this gelator by H-bonding, $\pi-\pi$ stacking and van der Waals interactions led to $\mathrm{J}$-aggregation which resulted in aggregation-induced emission in the gel state.

The self-assembly and gelation of the tripodal cholamide (Fig. 11a) was further exploited for the synthesis of 1D inorganic nanostructures. ${ }^{[53]}$ Nanotubes of inorganic oxides and sulphates have been reported using the tripodal cholamide-based hydrogel (Fig. 11b) as a template. ${ }^{[53 a]}$ Organic inorganic composite materials were obtained by the self-assembly of 2,3-didecyloxy-anthracene with AuNP ${ }^{54]}$ and $\mathrm{ZnO}$ NP. ${ }^{[55]}$ Syntheses of various nanotubes and nanorods such as $\mathrm{CdS}$ (Fig. 11c), $\mathrm{ZnS}$, and $\mathrm{CuS}$ have been established by using this hydrogel template approach. Steroidcapped NPs were stabilized by bile acidbased gelators due to the supramolecular interaction between the steroid units present both on the NP as well as on the gelator. ${ }^{[56]}$

Bannerjee et al. have reported synthetic tripeptide (with redox active chemical entities) based smart organogels for the in situ formation and stabilization of gold and silver nanoparticles within the supramolecular organogel networks (Fig. 12). ${ }^{[57]}$ These tripeptides contain redox active tyrosine residues, which have been utilized to reduce $\mathrm{Ag}(\mathrm{I})$ and $\mathrm{Au}(\mathrm{III})$ salts to generate $\mathrm{Ag}$ and Au nanoparticles in the gel phase without the presence of any external reducing and stabilizing agents. The TEM image of the hybrid gel in Fig. 12d indicates the formation of gold nanoparticles along the gel nanofibers. Peptide- and amino acidbased hydrogels have been used for the in situ generation of fluorescent silver nanoclusters at room temperature in presence of sunlight by using a 'green chemical' approach. ${ }^{58]}$ Fmoc-protected L-phenylalanine and the dipeptide Fmoc-Val-Asp-OH, individually form a transparent, stable hydrogel with a minimum gelation concentration of $0.1 \% \mathrm{w} / \mathrm{v}$ and $0.2 \% \mathrm{w} / \mathrm{v}$, respectively. The silver-ion-encapsulation of the hydrogels can spontaneously produce fluorescent silver nanoclusters under sunlight at physiological pH (7.46). Tripeptide hydrogel based nanofibers for the immobilization of luminescent $\mathrm{CdS}$ nanoparticles (NPs) within the gel matrix and a definite array of $\mathrm{CdS}$ nanoparticles on the gelnanofibers has been reported. ${ }^{[59]}$ Stiffness of the oligopeptide-based hydrogel has been modulated by the incorporation of different sized Ag nanoparticles stabilized by various capping ligands. ${ }^{[60]}$ Recently, the Fmoc-Phe-OH hydrogel has been used to disperse functionalized single-walled carbon nanotubes $(f$-SWCNTs) within the gel phase to make a hybrid hydrogel at physiological $\mathrm{pH}$ and temperature. ${ }^{[61]}$ One of these peptide-based hydrogels (FmocTyr-Asp-OH) has been utilized for the suc-

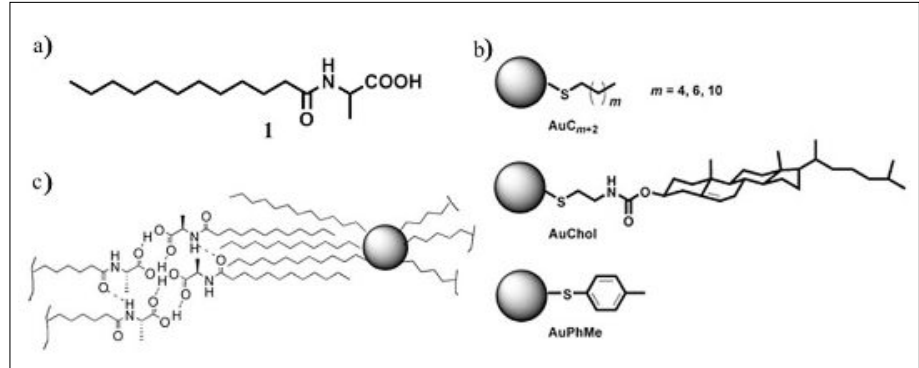

Fig. 10. (a) Chemical structure of the gelator molecule 1. (b) Capped AuNPs with various end groups. (c) Schematic representation of interaction of gelator 1 with capped AuNP's in gel state. Reprinted with permission from ref. [51a]. Copyright (2006) Wiley-VCH Verlag GmbH \& Co. KGaA, Weinheim.

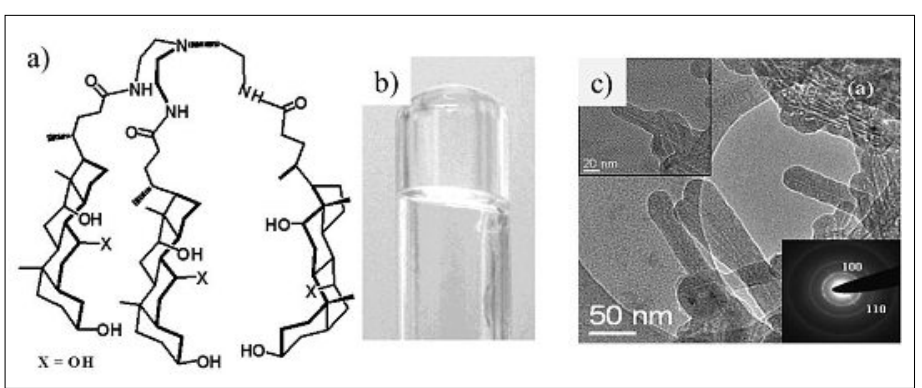

Fig. 11. (a) Chemical structure of tripodal cholic acid. (b) Photograph of transparent hydrogel of tripodal cholamide. Reprinted with permission from ref. [7]. Copyright (2004) American Chemical Society. (c) TEM image of CdS nanotubes obtained after the removal of hydrogel template. Reprinted from ref. [53b]. Copyright (2006) with permission from Elsevier. 
cessful incorporation of reduced graphene oxide (RGO) into the hydrogel to make a well-dispersed RGO-containing stable hybrid hydrogel.[62] This study demonstrates that RGO is stabilized within the peptidebased hydrogel system without the help of any external stabilizing agent. Rheological studies suggest the formation of a more rigid and 'solid-like' hybrid hydrogel after the incorporation of reduced graphene oxide into the peptide hydrogel.[62] Banerjee and coworkers have also made amino acidbased functional gels which have been utilized for photo-switching material[ ${ }^{[61]}$ and oil spill recovery. ${ }^{[63]}$

\subsection{Metallogels}

Subsequent to the success with organic salt-based gelators, Dastidar et al. reported ligand-based $\mathrm{Cu}^{\mathrm{II}} / \mathrm{Co}^{\mathrm{II}}$ coordination polymers which formed gels as well as crystals under different reaction conditions. These coordination polymers were derived from two bis-pyridyl-bis-amide ligands and various dicarboxylates. ${ }^{[64]}$ In addition, a series of $\mathrm{Zn}^{\mathrm{II}}$-based coordination polymeric gels were also reported.[65] Very recently, a strategy to separate $\mathrm{SO}_{4}^{2-}$ anion from a mixture of anions $\left(\mathrm{SO}_{4}^{2-}\right.$, $\mathrm{NO}_{3}^{-}, \mathrm{ClO}_{4}^{-}, \mathrm{CF}_{3} \mathrm{SO}_{3}^{-}$) using $\mathrm{Cu}^{\text {II }}$-based coordination polymers gels has been proposed.[66] $\mathrm{A} \mathrm{C}_{3}$-symmetric tris-amide-triscarboxylate ligand was used to obtain a set of four metallogels using various salts such as $\mathrm{Cd}\left(\mathrm{NO}_{3}\right)_{2}, \mathrm{Cu}\left(\mathrm{NO}_{3}\right)_{2}, \mathrm{Co}\left(\mathrm{NO}_{3}\right)_{2}$ and $\mathrm{Zn}\left(\mathrm{NO}_{3}\right)_{2} \cdot{ }^{[67]} \mathrm{A}$ series of bis-amides derived from $\mathrm{L}-(+)$-tartaric acid resulted in metallogels upon treatment with $\mathrm{Cu}^{\mathrm{II}} / \mathrm{Zn}^{\mathrm{II}}$ salts under suitable conditions. ${ }^{[68]}$

Biradha et al. have reported metalorganic gels based on bis(benzimidazole)based ligands with $\mathrm{Cd}$ (II) and $\mathrm{Cu}$ (II) halide salts. ${ }^{[69]}$ These gels exhibited dye and gas sorption properties. Bannerjee et al. have reported the first Ca-based metallogels and 3D MOFs by using 5-(1,2,4-triazoleyl) isophthalic acid (TIA). ${ }^{\text {70] }} \mathrm{Ca}$-based xerogels showed $20 \%$ higher $\mathrm{CO}_{2}$ uptake than the crystalline Ca-based MOF (Fig. 13). These metal organic materials have been used as catalysts for the hydrosilylation of benzaldehyde with diphenylsilane, where the gel phase showed higher catalytic activity in comparison to the crystalline or xerogel materials.

\subsection{Liquid Crystals and Polymer Composites}

Gelation-assisted trapping of fluorescent supramolecular architectures in a polystyrene (PS) film and its application in erasable thermal imaging has been reported. [71] OPV gelators are known to have thermally reversible fluorescence (blue to green upon sol-gel transition and vice versa). The gelator forms a gel in styrene with green emission which upon photopoly-

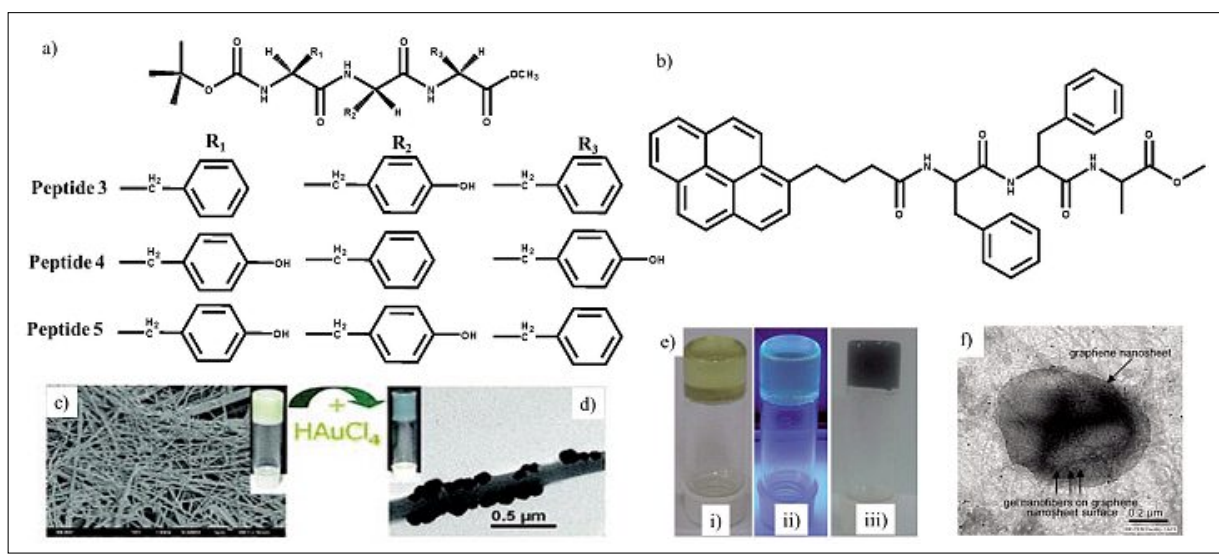

Fig. 12. Chemical structures of tripeptides (a) and pyrene-conjugated gelator peptide (b). TEM images of the gel nanofiber before (c) and after (d) the in situ formation of nanoparticle, black dots indicate the Au nanoparticles. Reproduced with permission from ref. [57] with permission from the Royal Society of Chemistry (C) 2007. (e) Photographs of organogels i) under exposure to daylight, ii) under exposure to UV light (excitation at $365 \mathrm{~nm}$ ), and iii) graphene-containing hybrid organogel under exposure to daylight. (f) TEM image of the hybrid organogel. Reprinted with permission from ref. [62b]. Copyright (2011) Wiley-VCH Verlag GmbH \& Co. KGaA, Weinheim.

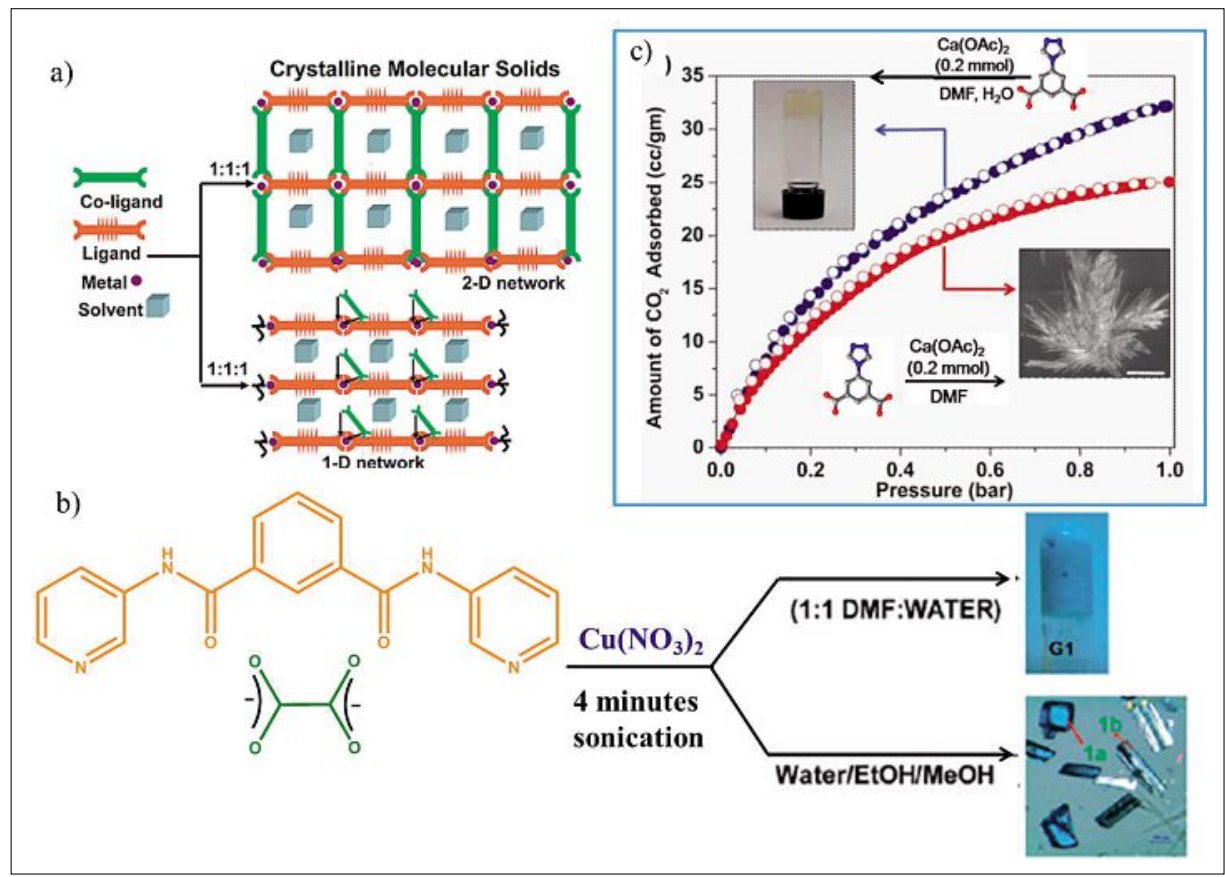

Fig. 13. (a) Schematic representation of 1D and 2D coordination polymeric networks. (b) Sonication induced gelation of ligand with dicarboxylates and metal ion. Reprinted with permission from ref. [63]. Copyright (2010) American Chemical Society. (c) $\mathrm{CO}_{2}$ adsorption isotherms below 1.0 bar for Ca-5TIA-xerogel (blue) and Ca-5TIA-MOF (red) at $298 \mathrm{~K}$. Reproduced with permission from ref. [70] with permission from the Royal Society of Chemistry; @ 2012.

merization resulted in the formation of the gelator entrapped polymer. A film of the OPV-PS polymer composite was prepared by dropcasting a chloroform solution of the same. By heating above $T_{\mathrm{g}}$, the OPV-PS blend became soft and the thermal energy induced breakage of the initially formed OPV self-assembly (responsible for the green emission) into individual molecules with strong blue emission. Upon exposure to chloroform vapors, the film is softened thereby allowing the reassembly of the OPV molecules, regenerating the green emission. Thus, the mechanism of the rewritable imaging has been explained as a reversible self-assembly of the OPVs in the PS film. Since the fluorescence of the unimaged area was weak and that of the imaged area was strong, high-contrast imaging could be achieved. Aligned photoluminescent LC physical gels were obtained by the simple mixing of an OPV-based gelator with nematic and smectic liquid crystals. ${ }^{\text {[2] }}$ When self-assembly of OPV occurs in the LC phase, aligned fibers were formed through the template effects of the liquid crystals. The alignment of $\pi$-conjugated OPV gelators in nematic liquid crystals was found to be different from normal gelators. 


\subsection{Donor-Acceptor-based Gels}

Ghosh and coworkers have reported structure-property relationships in the gelation of electron-rich dialkoxy-naphthalene (DAN) and naphthalene-diimide (NDI) building blocks. ${ }^{[73]}$ They have also studied self-assembly and gelation of electron-rich, dialkoxynaphthalene (DAN). Unlike NDI-chromophores, long rangeordered arrangement of DAN is difficult due to the strong electronic repulsion between the adjacent donor chromophores. The bis-urea (DAN-U) and the bis-amide (DAN-A) functionalized DAN-derivatives (Fig. 14), which differ only in the nature of the self-complementary H-bonding functionality, showed similar spectral behavior in a good solvent like THF in which they were molecularly dissolved. However, in a non-polar solvent like $\mathrm{MCH}$, the nature of chromophoric arrangement of DAN-U and DAN-A was different as observed from the absorption and the emission spectra of the two chromophores. The J-aggregating DAN-U formed gels in various organic solvents, both polar as well as non-polar with very low critical gelator concentration (CGC) values. Contrastingly, the $\mathrm{H}$-aggregating DAN-A formed gel in a limited number of non-polar solvents with comparatively higher CGC values. DAN-A formed highly entangled flexible fibers owing to the weaker $\mathrm{H}$-bonding between the amide groups and was more effective in solvent trapping to produce better gels. On the other hand, stronger H-bonding among the urea functionalities led to more rigid rodlike fibers which could not form a network structure required for the efficient gelation. Interestingly, the H-bond mediated gelation of the donor (DAN-2) and the acceptor (NDI-2) chromophores exhibited self-sorted assembly. ${ }^{[74]}$

\subsection{Fluorescence Modulation and Energy Transfer in Gel Scaffolds}

OPV gelators exhibit significant red shift of the emission during the gelation. This property has been shown to be due to the efficient energy migration between aggregates of different energy levels. ${ }^{\text {[75] }}$ Therefore, OPV gels are very good energy donor scaffolds for energy transfer to a suitable acceptor. This hypothesis was proved by energy transfer from OPV gels to entrapped Rhodamine B as the acceptor.[76] Fluorescence resonance energy transfer (FRET) was found to be more efficient in a xerogel film when compared to the selfassembled gels obtained from dodecane or cyclohexane. Thus, xerogels of OPVs are unique examples for nanostructured $\pi$-conjugated self-assemblies, which are efficient energy donor scaffolds for thermally gated FRET processes and hence became an entry to a novel class of functional supramolecular materials. ${ }^{[77]}$ However,

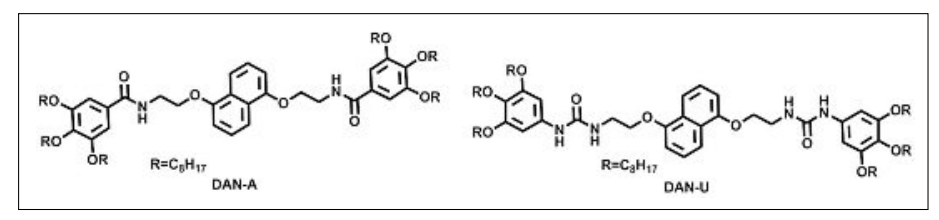

Fig. 14. Chemical structures of DAN-U and DAN-A

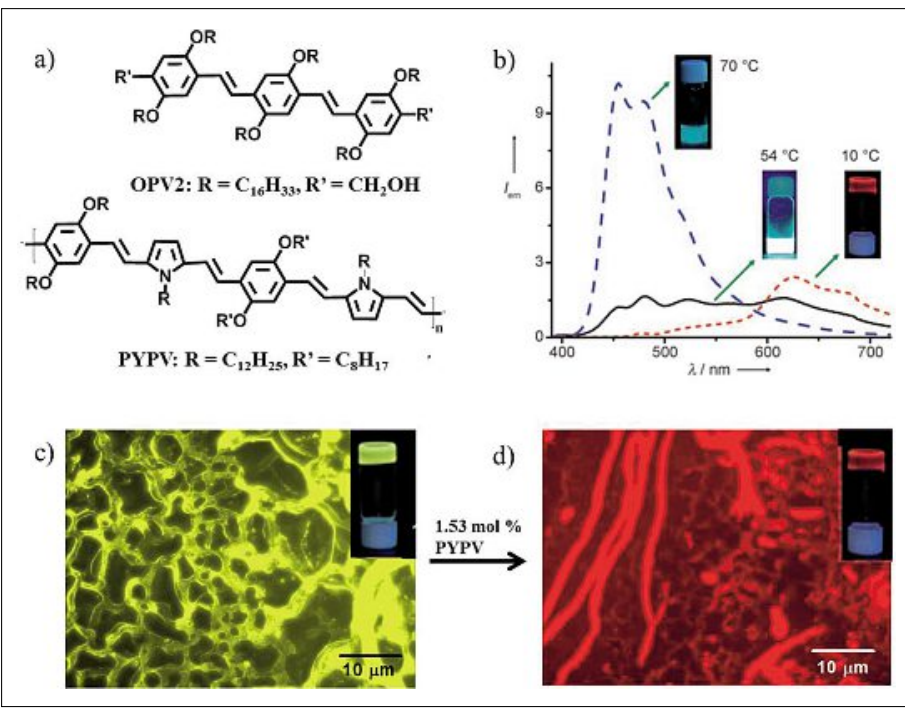

Fig. 15. (a) Chemical structures of OPV2 and PYPV (b) Temperaturedependence of the energy transfer. Fluorescence microscopy images of the drop-cast OPV2 before (c) and after (d) addition of PYPV cyclohexane gel. Reprinted with permission from ref. [80]. Copyright (2007) Wiley-VCH Verlag GmbH \& Co. KGaA, Weinheim.

the poor compatibility of Rhodamine B and OPV resulted in poor overall energy transfer efficiency. This problem could be solved by choosing compatible energy acceptors. ${ }^{[78]}$ By choosing suitable acceptors, the emission color could be continuously shifted towards the longer wavelengths. ${ }^{[78]}$

The energy transfer efficiency of OPV gels was found to be high when a short conjugated oligomer PYPV was used as the acceptor in small quantities (Fig. 15). ${ }^{[80]}$ Such efficient energy transfer at the gel state of the donor molecules with a small percentage of the acceptor molecule indicates efficient energy funneling from a distance beyond the normal Föster radius, indicating cascade energy migration between the aggregated gelator molecules. The energy transfer properties could also be controlled by the structural modification of OPV gelators as demonstrated with the mono- and bis-cholesterol functionalized OPV gelators. ${ }^{[12]}$ In the case of the mono-cholesterol derived OPV gelator, a red emission was obtained whereas in the case of the bis-cholesterol derived OPV gelator, a white emission was observed, when PYPV was used as the acceptor in the gel state.

Maitra et al. have reported a luminescent hydrogel, which can be used as an efficient matrix for photosensitized energy transfer between Eu(III) and pyrene.[81] Inexpensive and simple sensors for enzymes as well as metal ions were also constructed from lanthanide-based supramolecular systems. ${ }^{[82]}$ Self-assembly of a new class of amphiphilic phenylenevinylene bis- $N$-alkyl pyridinium salts (PPV) with end aliphatic hydrocarbon chains of vari- ous lengths was reported.[83] Temperaturedependent emission of these PPVs led to white light emission between $25-30{ }^{\circ} \mathrm{C}$ in solution. It was found that viscoelastic and electrical conductivity behavior are related to the terminal aliphatic chain length. Bhattacharya and coworkers have reported energy transfer in a self-assembled OPV derivative which acts as donor (D), with various acceptor molecules, such as a different OPV derivative (A), anthracene $(\mathbf{N})$, and rhodamine (R) 6G. ${ }^{\left[{ }^{[8]} \text { Self-assembly }\right.}$ of all four chromophores $(\mathbf{D}, \mathbf{A}, \mathbf{N}$, and $\mathbf{R})$ in a particular fashion was the key process in this cascade of energy transfer assemblies containing different luminescent compounds. The light harvesting behavior was based on the individual chromophores as removal of any one of the molecule from the assembly led to the absence of energy transfer process.

George et al. have reported the design of the noncovalent self-assembly of chromophores in an organo-clay template resulting in the formation of fluorescent hybrid hydrogels and films. ${ }^{[85]}$ These clay-dye hybrids act as novel supramolecular scaffolds for light-harvesting due to the spatial organization of donor and acceptor molecules to promote Förster resonance energy transfer. The authors used tetracarboxylates of coronene (CS, blue emission) and perylene (PS, green emission) as the donor-acceptor molecules, because of their high solubility in water. The negatively charged carboxylate groups of these dyes interact electrostatically with the positively charged amino clay. The bifunctional nature of both organic and inorganic building blocks further facilitated a 
three-dimensional self-assembly in aqueous solution, thus resulting in non-covalent hybrid materials. Efficient energy transfer from CS to PS was observed in highly crosslinked hybrids in solution when the former was excited at $350 \mathrm{~nm}$, resulting in an intense green emission from perylene chromophores. Energy transfer studies revealed complete quenching (100\% FRET efficiency) of donor emission (CS) even with small amount of PS ( $<10 \mathrm{~mol} \%)$, owing to very efficient energy transfer. These light-harvesting soft materials could be successfully processed onto solid substrates by spin/drop casting and the resultant transparent films showed significant donor quenching, even with less than $1 \%$ acceptor loading. Furthermore, the energy transfer by co-assembly increases the fluorescence quantum yield of the hybrid gels and films, as the individually assembled dye/clay hybrids were virtually non-fluorescent. In addition, the emission of the hybrid gels was tuned from green to yellow by increasing the acceptor dye concentration, which resulted in the energy transfer from CS to PS aggregates (Fig. 16). [86]

\section{Recent Developments}

During the period 2011-2012, several interesting studies with new gelators were reported from the groups of young researchers. These studies include sugarbased gelators, C-T based gelators etc. In addition, more insights on the gelation of $\pi$-systems and their application in sensing have been reported; some of which are highlighted below.

\subsection{Insight into the Gelation of $\pi$-Systems}

Guenet and coworkers carried out a series of experiments to understand the role of functional groups in OPVs and the role of solvents in the gelation process. ${ }^{[87]}$ Such experiments detailed the dramatic effect of the solvent on the molecular structure and on the morphology of the organogels. ${ }^{[87 b, c]}$ These investigations revealed that the selfassembly of OPV-based gelators can be extremely sensitive to the chemical structure of the end groups located on the backbone. These end groups also play a decisive role in the morphology and the molecular level structure of OPV-based organogelators. The gel thermodynamics ( $\mathrm{T}-\mathrm{C}$ phase diagram), morphologies, molecular order, and optical properties differ considerably with the solvent type. ${ }^{[87 \mathrm{~d}]}$ These observations were explained by considering the interaction of the solvent with the different moieties of the OPV molecule. Govindaraju et al. demonstrated intermolecular hydrogen bond-directed self-assembly of cyclic dipeptide derivatives into nanofibers, na-

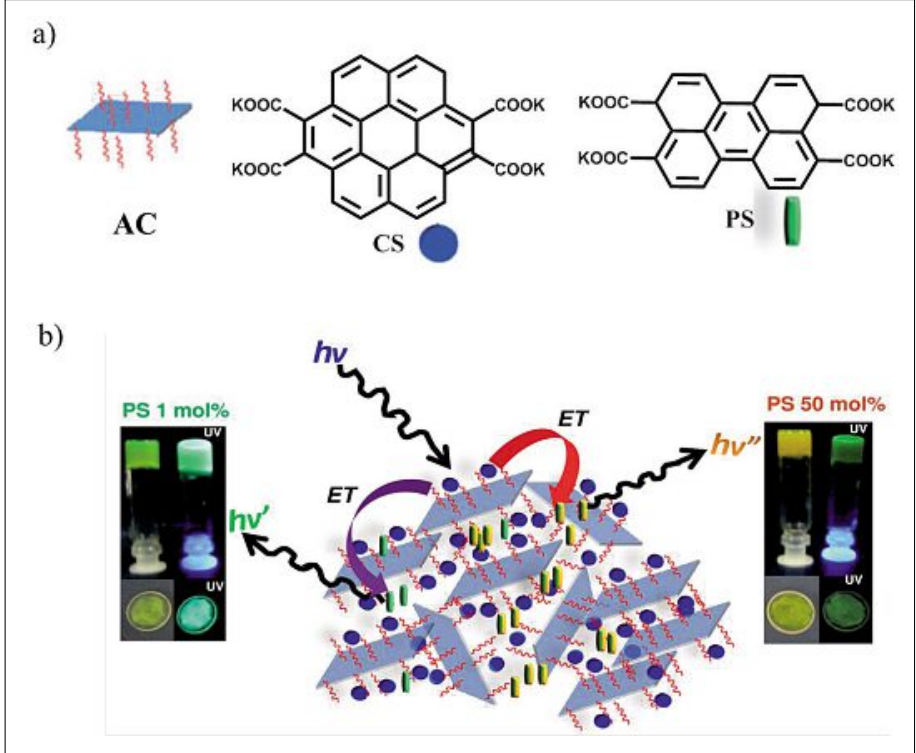

Fig. 16. (a) Chemical structures of a single layer of aminoclay (AC) and anionic dyes (CS and PS) (b) photographs and schematic representation of the energy transfer from CS to isolated (1 mol\%) and aggregated (50 mol\%) PS in clay-dye hybrid gels and films. (Pictures received from original author.)

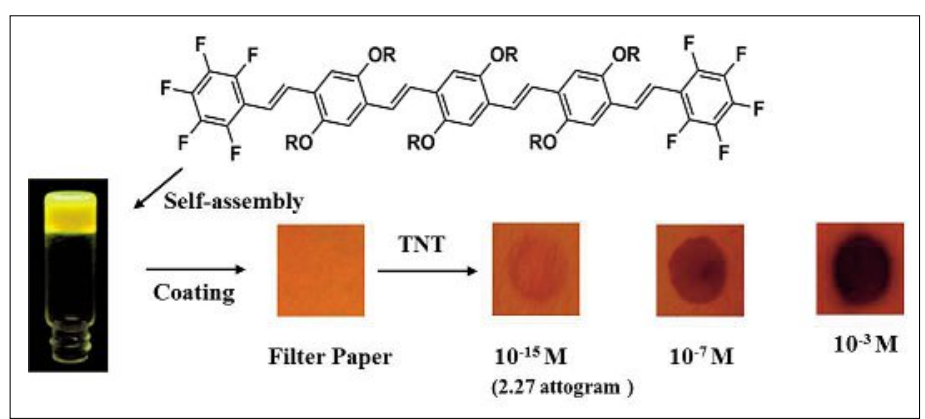

Fig. 17. Chemical structure of OPVPF. Demonstration of self-assembly and TNT detection using the gel filter paper strip. Reprinted with permission from ref. [91]. Copyright (2012) American Chemical Society.

noribbons and their subsequent gelation. ${ }^{[88]}$ These gels can be used as entrapping agents, drug delivery systems and also as thermoresponsive soft materials. Pramanik et al. reported the self-assembly of modified tripeptides into various micro- and nanostructured materials such as nanovesicles, nanotubes, giant microvesicles, macroporous vesicular structures including macroporous films, macro- and mesoporous materials, and organogels by means of fine-tuning of the solvent polarity. ${ }^{[89]}$ Ajayaghosh et al. demonstrated the influence of the length of the oligomer in a linear $\pi$-system on molecule-molecule and molecule-substrate interactions, which could influence the morphological features of the self-assembly. More importantly, this study provides an insight into the surface and 'ambient-adaptable' self-assembly of extended $\pi$-systems, thereby allowing access to a variety of aesthetically appealing super-structures. ${ }^{[90]}$

\subsection{Gels as Sensors}

Fluorescence is one of the most sensitive properties of $\pi$-gels. The reversible modulation of fluorescence has been exploited for the sensing of explosives. For example, attogram level detection of TNT is possible with an OPV gelator (OPVPF), conjugated with pentafluoroarene end groups. ${ }^{[91]}$ The arene-perfluoroarene interaction facilitates a brick-wall type assembly of the gelator in which the electron-deficient TNT could be entrapped resulting in the quenching of the fluorescence. Filter papers coated with OPVPF gels are found useful for the contact mode sensing of TNT as low as $\sim 12$ $\mathrm{ag} / \mathrm{cm}^{2}$ (Fig. 17). Interestingly, the gelator molecules in solution could not efficiently detect TNT, revealing the role of the molecular assembly in the sensing ability. Bhalla et al. have synthesized triphenylene-based discotic liquid crystal derivatives bearing 1,2,3-triazole groups which resulted in the stabilization of columnar mesophases down to room temperature, leading to the formation of organogels in cyclohexane and mixed solvents such as hexane and dichloromethane. The strong emission of the gelator in its nonaggregated form makes it a promising fluorescence sensory material for nitroaromatic compounds. ${ }^{[92]}$

\subsection{Oil Spill Recovery and Soft Optical Device Development}

Sureshan et al. have synthesized organogelators based on D-mannitol, which can gelate nonpolar solvents and oils. ${ }^{[93]}$ Mannitol-based phase selective supergelators have been used to solidify oil from a mixture of oil and water at low concentrations of the gelator. Particularly, gelation of oil in a biphasic mixture with fresh water, $3.5 \% \mathrm{NaCl}$ solution and seawater revealed 
that the gelation efficacy was the same in all these biphasic systems. The isolated oil could be recovered by melting the gel by heating and distilling. ${ }^{[93 \mathrm{~b}]}$ Gelation experiments of the two mannitol-based gelators (Fig. 18a) with solvents and oils resulted in gels with alkane solvents at very low concentrations with CGCs as low as $0.2 \mathrm{wt} \%$, moving them to the category of supergelators. These gels are sufficiently strong and are stable for months without losing their transparency, volume, and shape. Also these gels have shown self-healing and shape molding properties. Since these oilbased gels were strong, highly transparent, self-healing, and have glass-like refractive indices, it could be exploited for making soft optical devices. Gels of these derivatives in hydrocarbon solvents or oils show a high transmittance to visible region and weak to UV region, resulted as a soft UV filter. ${ }^{[93 a]}$

\subsection{Recent Developments in Self- sorting Gels}

Self-sorting is a phenomenon observed in the case of certain donor-acceptor combinations. When such molecules are allowed to self-assemble, they form self-sorted assemblies of the individual molecules. Ghosh et al. have reported a series of bis-amide functionalized NDIbased building blocks (Fig. 19) with varying numbers of methylene groups between the chromophore and amide functional groups and studied the effect of the spacer length on self-assembly and gelation. ${ }^{94]}$ Spectroscopic studies revealed formation of self-assembled structure in relatively non-polar solvents due to the synergistic effect of $\pi-\pi$ stacking, hydrogen bonding and hydrophobic interactions. Propensity towards self-assembly was found to follow the order NDI-0 $>>$ NDI-2 $\sim$ NDI-3 $>$ NDI-4 from solvent and temperaturevariable UV/Vis spectroscopic studies.

NDI-2 and DAN-2, in which the distance between the two amide groups was different, when mixed in a non-polar solvent $(\mathrm{MCH})$ formed a segregated assembly leading to yellow gels. ${ }^{\left[{ }^{95]}\right.}$ In aggregated assembly, all the amide groups could be involved in $\mathrm{H}$-bonding interaction which was not possible in the alternate D-A stack due to geometrical constraint. Interestingly, DAN-4 and NDI-2 in which the amide group distances are comparable upon mixing gave a red gel due to the CT-inter-

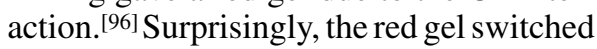
to a yellow one within a few hours due to the rearrangement of the donor and the acceptor molecules from the CT-state to the self-sorted state. As H-bonding is known to be stronger than $\pi$-stacking or CTinteraction, the initially formed kinetically controlled CT-gel was changed to a thermodynamically more stable self-sorted gel

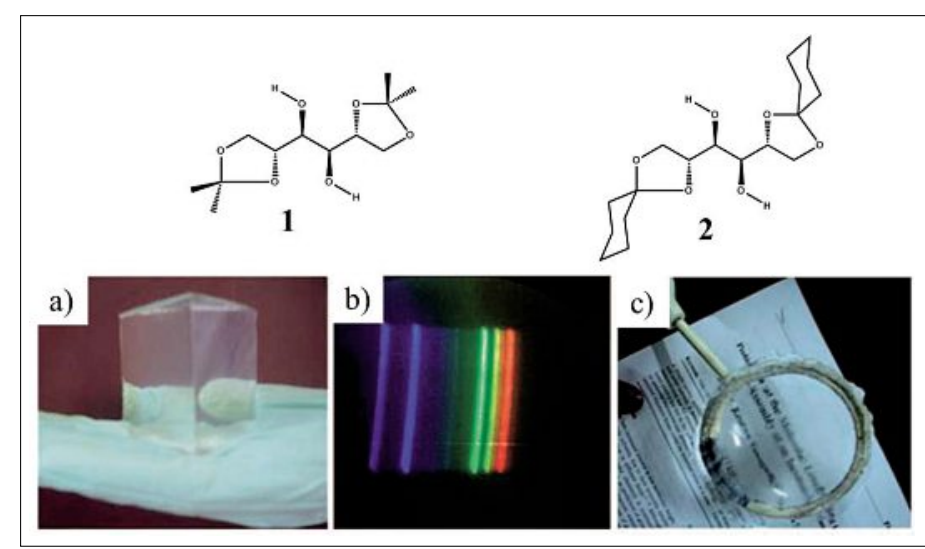

Fig. 18. Chemical structures of Dmannitol derivatives. (a) A gel prism made from pump oil gel of 2. (b) The diffraction pattern observed using the gel prism. (c) The double convex gel lens made from the pump oil gel of $\mathbf{2}$ supported on a metallic ring. Reprinted with permission from ref. [93a]. Copyright (2011) Wiley-VCH Verlag GmbH \& Co. KGaA, Weinheim.

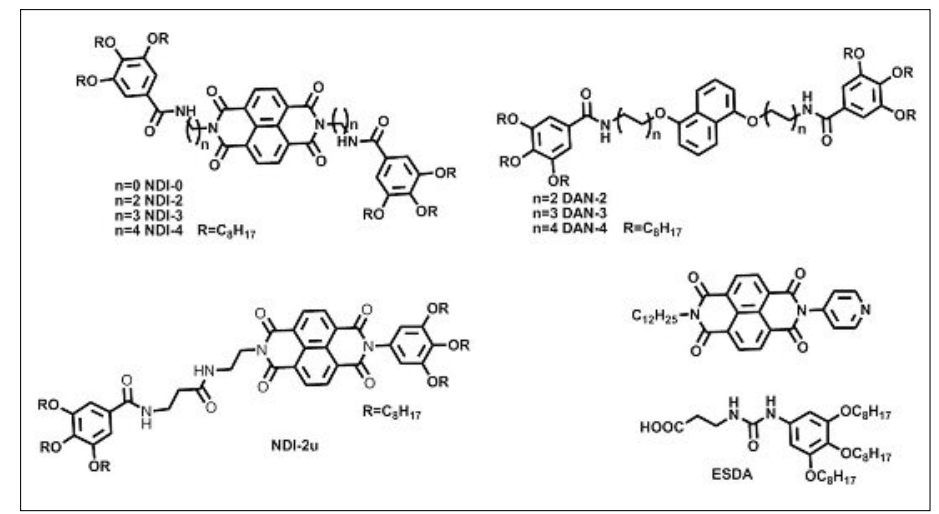

Fig. 19. Chemical structures of selfsorting gelators. by overcoming the weaker CT-interaction and the constrained $\mathrm{H}$-bonding in the alternate D-A stacking by the geometrically favored $\mathrm{H}$-bonding in the homoaggregates. To gain further insight into this unique supramolecular switching phenomenon in D-A mixed systems, co-assembly of various structurally related D and A chromophores were prepared by varying their spacer length. ${ }^{[97]}$ Gelation of NDI-2 with another structurally related donor molecule DAN-3 in tetrachloroethylene (TCE) revealed more stable alternate $\mathrm{D}-\mathrm{A}$ type stacking than NDI-2 + DAN-4 due to the presence of a lesser number of methylene units. Surprisingly in this case switching was also observed in TCE as the gelation ability of DAN-3 was found to be stronger than that of DAN-4. Aiming towards a more generalized strategy for self-sorting donor and acceptor gels, co-assembly of the unsymmetrical bis-amide functionalized building block NDI-2u with various symmetrical bis-amide substituted DANgelators was investigated. ${ }^{[98]}$ In all cases, self-sorted gelation was noted. It has been found that breaking the symmetry did not have any adverse effect on the self-assembly by comparing the gelation properties of NDI-2u and NDI-2 which showed even better thermal stability for the unsymmetrical gelator. ${ }^{[99]}$ Very recently a novel supramolecular strategy for externally induced gelation of NDI derivative using orthogonal H-bonding interaction has been reported. ${ }^{[100]} I-\mathrm{V}$ measurements showed superior conducting property for the fibrillar gels when compared to spherical assembly.

\subsection{Gels Based on Poly(aryl ether) Dendrons}

Prasad et al. have reported the synthesis, gelation and photophysical properties of luminescent $\mathrm{AB}_{3}$ type poly(aryl ether) dendrons without conventional gelation motifs such as peptides, long alkyl chains, steroidal groups etc. ${ }^{[101]} \pi-\pi$ Interaction is the major noncovalent force involved in the self-assembly and gelation, which was controlled by the polarity of the medium. The close packing of the poly(aryl ether) units enhances the feasibility of the excimer formation upon photo-excitation of the gel. These are examples of excimerbased fluorescent low molecular mass organogelators (LMOG) by poly(aryl ether) dendrons, in the absence of usual gelating motifs. These compounds prefer to form gels in solvent mixtures, rather than in pure solvents. Anthracene substituted poly(aryl ether) dendron derivatives, which have no hydrogen bonding sites, readily form entangled nano-fibrils in chloroform-methanol mixtures $(1: 3 \% \mathrm{v} / \mathrm{v})$. An important finding from Prasad and coworkers includes the design, synthesis and the photophysical properties of an acylhydrazonelinked anthracene derivative of $\mathrm{AB}_{3}$ and $\mathrm{AB}_{2}$ type poly(aryl ether) and its utility for the 'naked eye' detection of fluoride ions 
(as low as 0.1 equivalent with respect to the gelator concentration) through a reversible sol-gel transition which is associated with a color change from deep yellow to bright red (Fig. 20). ${ }^{[102]}$ This could be, presumably, through proton abstraction from the gel by the fluoride ion. Subsequently, the $\mathrm{H}$-bonding in the gel is weakened, leading to the gel-sol conversion. Another interesting observation is the solvent-controlled self-assembly of pyrene-cored poly(aryl ether) dendron derivatives that form in nano-sized vesicles, which further aggregate to micro-sized vesicles and finally turn to entangled fibrillar type arrangement in the gel phase. ${ }^{[103]}$ This gelator also has been demonstrated as an efficient system for the detection of fluoride ions by the solgel transition with an intense color change from yellow to red.

\subsection{Photoresponsive Gels and Nanostructures}

Organic supramolecular nanorods of an azobenzene-derived molecular (Fig. 21a) assembly were prepared without using templates, which can be assembled and disassembled by irradiation under light of appropriate wavelengths. ${ }^{[104]}$ This study illustrates the use of light as a tool to control the ripening of amorphous organic nanodots to micrometer-sized supramolecular rods. The low photoisomerization yield (cis-13\%) has become advantageous for the observed morphology transition. The nanodots could be regenerated by heating the nanorods to $60{ }^{\circ} \mathrm{C}$ followed by cooling at a rate of $1{ }^{\circ} \mathrm{C} / \mathrm{min}$ under irradiation with visible light. Recently, a phase-selective low-molecular weight photoswitchable sugar hybrid gelator has been reported that selectively gelates aromatic solvents. ${ }^{[105]}$ The trans form of the molecule which favors the long-range self-assembly leads to an entangled network structure that can form a gel in aromatic solvents. After UV irradiation the bundled fibers disintegrates to give short globular aggregates due to the trans-cis isomerization of the gelator molecules (Fig. 21b). The partial trans-cis isomerization occurs randomly at different parts of the fibers which allows the chopping of the fibers. In a recent report photoresponsive OPE gelators (Fig. 21c), functionalized with photochromic azo moieties are shown to undergo helicity inversion when irradiated at the aggregate melting temperature. This system is an example to demonstrate that the handedness of a photoresponsive supramolecular object can be biased with the action of light and heat, without changing the inherent molecular chirality of the individual building blocks. This study strengthens the general perception that in combination with other forces, light may help to determine parity violation in the symmetry of natural objects. ${ }^{[106]}$

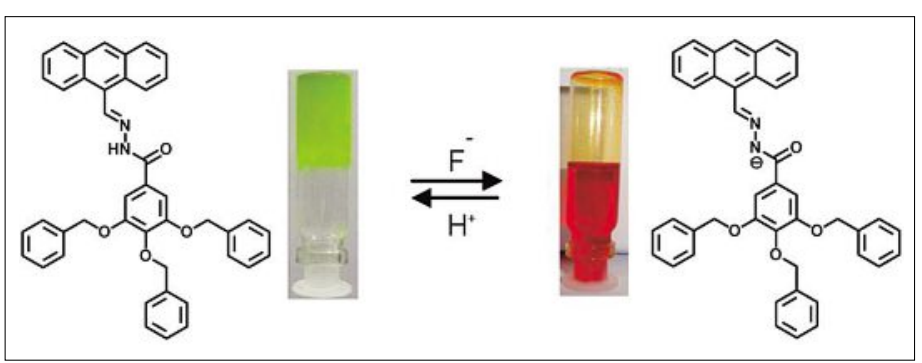

Fig. 20. Chemical structure of a trisbenzylether for fluoride ion detection. Reprinted with permission from ref. [102]. Copyright (2011) American Chemical Society.

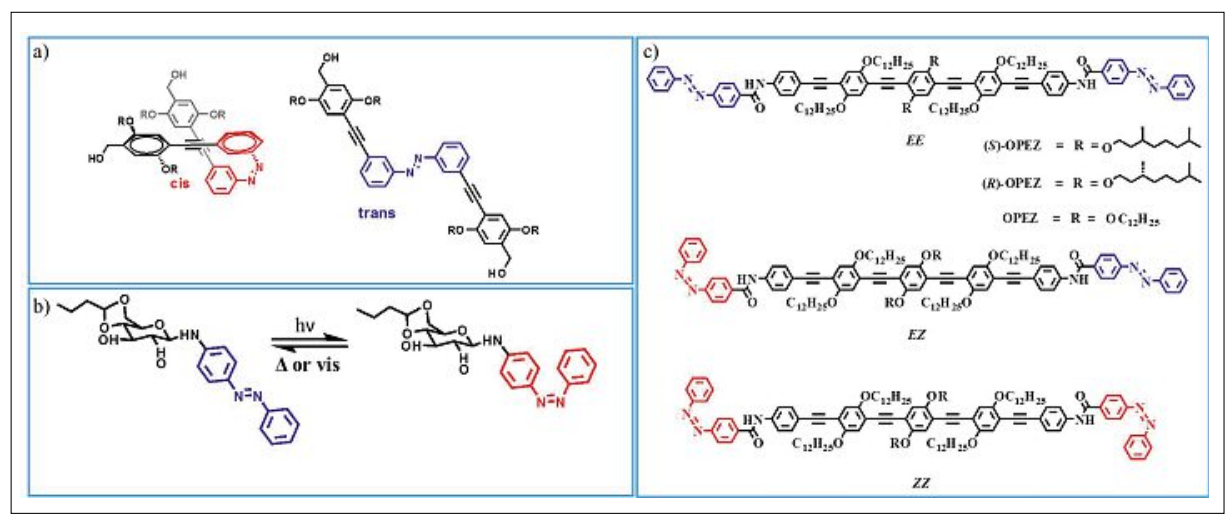

Fig. 21. Chemical structures of photoresponsive $\pi$-gelators.

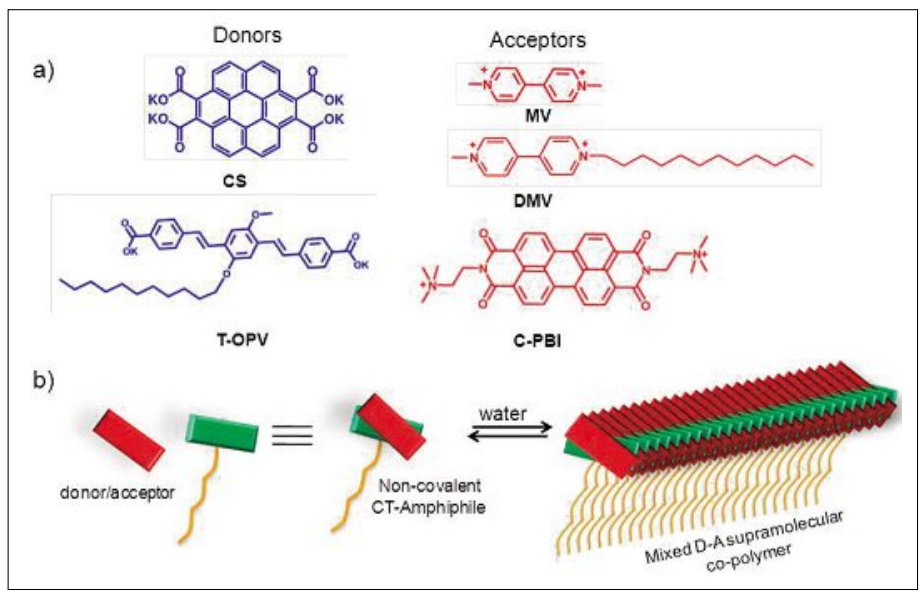

Fig. 22. (a) Molecular structures of donor and acceptor molecules. (b) Schematic representation of the non-covalent amphiphile and its self-assembly into 1D supramolecular structures. (Figures received from original author).

\subsection{Non-covalent Charge-transfer Amphiphilic Gelators}

George et al. have designed a donor-acceptor pair in such a way that the resultant CT-complex of these molecules resembles an amphiphile and facilitates 1D self-assembly in water through hydrophobic interactions (Fig. 22). ${ }^{[107]}$ This socalled 'non-covalent amphiphilic design' strategy was successfully demonstrated with structurally different donor-acceptor molecules. ${ }^{[108]}$ The first D-A pair that was investigated in this context was composed of coronene salt (CS) and methyl viologen (MV) as electron donor and acceptor respectively (Fig. 22a). This pair forms 1:1 CT-complex in water and the obtained CT-crystal was composed of 1D columns of alternatively arranged CS and MV molecules. As expected, this pair failed to form nanofibers in solution due to the absence of any flexible self-assembling groups and the rigid planar nature of both donor and acceptor molecules which led to the formation of crystals. However, after replacing one of the methyl group of MV with a dodecyl chain (DMV), the resultant CTcomplex in water showed the formation of high aspect ratio nano fibers. A deep red colored hydrogel was formed at a high concentration $(7.96 \mathrm{mM})$ of the CS-DMV complex which showed strong CT-band absorption at $500 \mathrm{~nm}$ with completely quenched donor fluorescence (Fig. 23a). A field effect transistor (FET) was fabricated by drop casting the aqueous solution of CS-DMV nanofibers across the sourcedrain electrodes on $\mathrm{SiO}_{2} / \mathrm{Si}$ substrate (Fig 23b,c). ${ }^{[109]}$ The observed field effect mobility was as high as $4.4 \mathrm{~cm}^{2} / \mathrm{Vs}$ which is the highest among organic polymers and small molecule-based self-assembled nanowires reported so far. Interestingly, these nanofibers displayed self-repairing FET characteristics. For example, the as-made device consisting of $12 \mathrm{CS}-\mathrm{DMV}$ fibers with a 

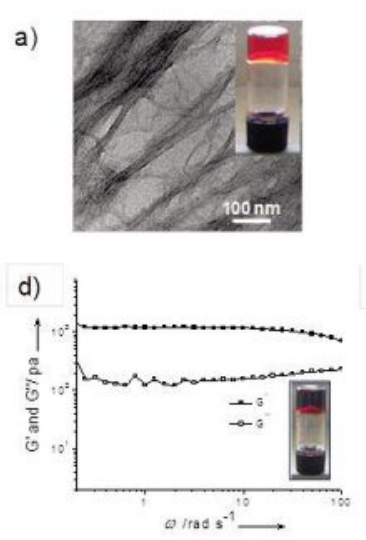

b)

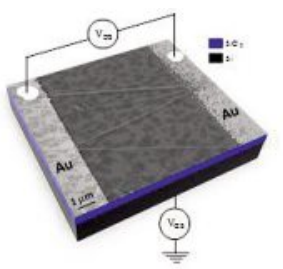

e)

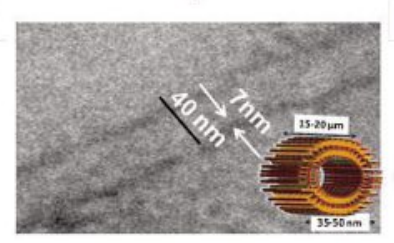

c)

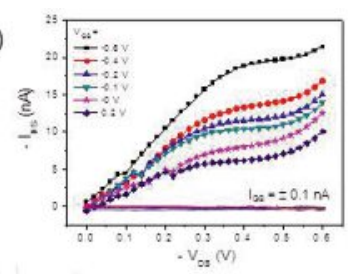

f)

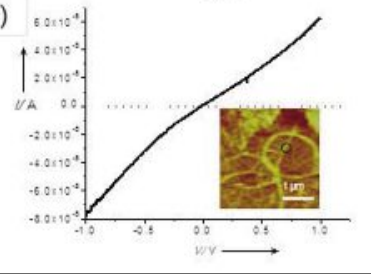

Fig. 23. (a) TEM images of cylindrical micelle formed from CS-DMV (1:1) CT-complex and the photograph of CS-DMV hydrogel $(7.96 \mathrm{mM})$ is shown in the inset. (b) A SEM image of CTnanofibers between gold electrodes adapted in the schematic of the FET. (c) Output characteristics of the FET with five CS-DMV nanofibers (the measured diameters of the nanofibers are 200, 230, 330, 120 and $185 \mathrm{~nm}$ ). (d) Storage (G') and loss (G') modulus of the T-OPV:C-PBI gel measured as function of angular frequency $(\omega)$ at a constant strain of $1 \%$ (Photographs of the gel exhibiting self-standing behavior in inset). (e) TEM image and schematic representation (inset) of double walled nanotube formed from the T-OPV:C-PBI non-covalent amphiphile. (f) C-AFM I-V curves of nanotubes $(0.1 \mathrm{~mm})$ drop-cast on to the gold-coated glass substrate. The black circle in the inset image shows the nanotube on which the $\mathrm{I}-\mathrm{V}$ measurements were performed. (Figures received from original author).

channel width of $2.4 \mu \mathrm{m}$ showed a mobility of $1.33 \mathrm{~cm}^{2} / \mathrm{Vs}$ which decreased to 0.77 $\mathrm{cm}^{2} / \mathrm{Vs}$ after seven days. The original mobility was regained by treating the nano fibers on the device with a drop of water. The self-repairing ability of the present system could be attributed to the regeneration of the tight D-A packing and stands out when compared to conventional polymer FETs which fail to regain their electrical properties once the device loses its function.

George and coworkers have further extended this non-covalent amphiphilic design principle to make extended supramolecular alternate co-polymers of wellknown p-type and n-type semiconductors such as oligo(phenylenevinylene) (OPV) and perylenebisimide (PBI) respectively in water (Fig. 22). ${ }^{[108]}$ At higher concentrations (3.3 mM), these complexes form dark wine colored hydrogels with excellent mechanical (elastic) properties. The value of storage modulus $\mathrm{G}^{\prime}$ was $\sim 1000 \mathrm{~Pa}$ and the ratio of $\mathrm{G}^{\prime}$ to $\mathrm{G}^{\prime \prime}(\sim 8)$ remain unaffected up to an angular frequency of $70 \mathrm{rads}^{-1}$ owing to good mechanical stability and the elastic nature of these gels (Fig. 23d).

Detailed microscopic analysis of these systems at $0.1 \mathrm{mM}$ concentration in water showed that T-OPV forms cylindrical micelles of $3.5 \mathrm{~nm}$ and C-PBI forms micro rods whereas their 1:1 complex forms double-walled nanotubes which was distinct from the individual donor and acceptor morphologies (Fig. 23e). The formation of the tubular morphology for this CT-pair was mainly due to the wedge shape of the resultant CT-amphiphile which was different from the linear rod-coil amphiphile formed from CS-DMV pair. The observed conductivity for fibers having an average height of $35 \mathrm{~nm}$ was found to be $0.02 \mathrm{Scm}^{-1}$, and it falls in the highest values reported so far for a small molecule-based self-assembled nanostructure without external doping (Fig. 23f).

\section{Conclusions}

Even though a large number of hydrogelators have found various applications, organogelators have not found a breakthrough application as yet. Fluorescent $\pi$-gelators are expected to find application as sensors and security labels. It is worth trying to use some of the fluorescent gelators for the detection of volatile organic compounds (VOCs) which are formed in the human body as a consequence of certain diseases, which will help the early diagnosis of diseases. Another potential application of $\pi$-gelators is in organic electronic devices, particularly in organic bulk heterojunction photovoltaic devices. However, there is long way to go in terms of efficiency and stability of these materials for a real application. The future directions in this area of research should address some of these challenging issues.

\section{Acknowledgements}

We thank several of the authors whose work is described here for their inputs. A. A. is thankful to the Dept. Atomic Energy for financial support under the DAE-SRC Outstanding Researcher Award. K.K and R. M thank CSIR for research fellowship.

Received: January 7, 2013
[1] a) R. Balasubramanian, P. Rao, U. Maitra, Chem. Commun. 1999, 2353; b) N. M. Sangeetha, R. Balasubramanian, U. Maitra, S. Ghosh, A. R. Raju, Langmuir 2002, 18, 7154; c) S. Mukhopadhyay, G. Krishnamoorthy, U. Maitra, J. Phys. Chem. B 2003, 107, 2189; d) U. Maitra, P. Babu, Steroids 2003, 68, 459; e) S. Mukhopadhyay, U. Maitra, Curr. Sci. 2004 . 87, 1666; f) N. M. Sangeetha, S. Bhat, A. R. Choudhury, U. Maitra, P. Terech, J. Phys. Chem. B 2004, 108, 16056; g) P. Babu, D. Chopra, T. N. G. Row, U. Maitra, Org. Biomol. Chem. 2005, 3, 3695 .

[2] U. Maitra, P. V. Kumar, N. Chandra, L. J. D'Souza, M. D. Prasanna, A. R. Raju, Chem. Commun. 1999, 595.

[3] P. Babu, N. M. Sangeetha, P. Vijaykumar, U. Maitra, K. Rissanen, A. R. Raju, Chem.-Eur. J. 2003, 9, 1922.

[4] U. Maitra, V. K. Potluri, N. M. Sangeetha, P. Babu, A. R. Raju, Tetrahedron-Asymmetry 2001, 12, 477.

[5] P. Terech, N. M. Sangeetha, B. Deme, U. Maitra, J. Phys. Chem. B 2005, 109, 12270.

[6] a) U. Maitra, S. Mukhopadhyay, A. Sarkar, P. Rao, S. S. Indi, Angew. Chem. Int. Ed. 2001, 40, 2281; b) Nonappa, U. Maitra, Soft Matter 2007 3, 1428; c) P. Terech, U. Maitra, J. Phys. Chem. B 2008, 112, 13483.

[7] S. Mukhopadhyay, U. Maitra, Ira, G. Krishnamoorthy, J. Schmidt, Y. Talmon, J. Am. Chem. Soc. 2004, 126, 15905.

[8] S. Bhattacharya, Y. K. Ghosh, Chem. Commun. 2001, 185 .

[9] a) A. Ajayaghosh, S. J. George, J. Am. Chem. Soc. 2001, 123, 5148; b) S. J. George, A. Ajayaghosh, Chem.-Eur. J. 2005, 11, 3217.

[10] S. J. George, A. Ajayaghosh, P. Jonkheijm, A P. H. J. Schenning, E. W. Meijer, Angew. Chem. Int. Ed. 2004, 43, 3422.

[11] A. Ajayaghosh, R. Varghese, S. J. George, C. Vijayakumar, Angew. Chem. Int. Ed. 2006, 45, 1834.

[12] A. Ajayaghosh, C. Vijayakumar, R. Varghese, S. J. George, Angew. Chem. Int. Ed. 2006, 45, 456.

[13] S. S. Babu, S. Mahesh, K. K. Kartha, A. Ajayaghosh, Chem. Asian J. 2009, 4, 824.

[14] A. Ajayaghosh, R. Varghese, V. K. Praveen, S. Mahesh, Angew. Chem. Int. Ed. 2006, 45, 3261.

[15] A. Ajayaghosh, R. Varghese, S. Mahesh, V. K. Praveen, Angew. Chem. Int. Ed. 2006, 45, 7729.

[16] A. Ajayaghosh, S. J. George, A. P. H. J. Schenning, Top. Curr. Chem. 2005, 258, 83.

[17] S. Yagai, S. Mahesh, Y. Kikkawa, K. Unoike, T. Karatsu, A. Kitamura, A. Ajayaghosh, Angew. Chem. Int. Ed. 2008, 47, 4691.

[18] a) S. Yagai, S. Kubota, H. Saito, K. Unoike, T. Karatsu, A. Kitamura, A. Ajayaghosh, M. Kanesato, Y. Kikkawa, J. Am. Chem. Soc. 2009, 131, 5408; b) S. Yagai, H. Aonuma, Y. Kikkawa, S. Kubota, T. Karatsu, A. Kitamura, S. Mahesh, A. Ajayaghosh, Chem.-Eur. J. 2010, 16, 8652.

[19] T. Tazawa, S. Yagai, Y. Kikkawa, T. Karatsu, A. Kitamura, A. Ajayaghosh, Chem. Commun. 2010, 46, 1076

[20] S. Mahesh, R. Thirumalai, S. Yagai, A. Kitamura, A. Ajayaghosh, Chem. Commun. 2009, 5984.

[21] a) S. Manna, A. Saha, A. K. Nandi, Chem. Commun. 2006, 4285; b) A. Saha, S. Manna, A. K. Nandi, Langmuir 2007, 23, 13126; c) A. Saha, S. Manna, A. K. Nandi, Chem. Commun. 2008, 3732; (d) A. Saha, B. Roy, A. Garai, A. K. Nandi, Langmuir 2009, 25, 8457; e) P. Bairi, B. Roy, A. K. Nandi, J. Phys. Chem. B 2010, 114, 11454.

[22] P. Bairi, B. Roy, A. K. Nandi, J. Mater. Chem. 2011, 21, 11747 .

[23] P. Dastidar, Chem. Soc. Rev. 2008, 37, 2699.

[24] A. Ballabh, D. R. Trivedi, P. Dastidar, Chem. Mater. 2003, 15, 2136. 
[25] a) D. R. Trivedi, A. Ballabh, P. Dastidar, Chem Mater. 2003, 15, 3971; b) D. R. Trivedi, A Ballabh, P. Dastidar, B. Ganguly, Chem.-Eur. J. 2004, 10, 5311; c) D. R. Trivedi, P. Dastidar, Cryst. Growth. Des. 2006, 6, 2114; d) D. R. Trivedi, P. Dastidar, Cryst. Growth. Des. 2006 6, 1022.

[26] P. Sahoo, R. Sankolli, H. Y. Lee, S. R. Raghavan, P. Dastidar, Chem.-Eur. J. 2012, 18, 8057.

[27] P. Sahoo, D. K. Kumar, D. R. Trivedi, P. Dastidar, Tetrahedron Lett. 2008, 49, 3052.

[28] T. K. Adalder, N. N. Adarsh, R. Sankolli, P. Dastidar, Beilstein J. Org. Chem. 2010, 6, 848

[29] D. R. Trivedi, P. Dastidar, Chem. Mater. 2006, 18,1470 .

[30] A. Ballabh, D. R. Trivedi, P. Dastidar, Org. Lett. 2006, 8,1271 .

[31] P. Sahoo, V. G. Puranik, A. K. Patra, P. U. Sastry, P. Dastidar, Soft Matter 2011, 7, 3634.

[32] P. Sahoo, I. Chakraborty, P. Dastidar, Soft Matter 2012, 8, 2595.

[33] P. Dastidar, S. Okabe, K. Nakano, K. Iida, M. Miyata, N. Tohnai, M. Shibayama, Chem. Mater. 2005, 17, 741.

[34] A. Ballabh, D. R. Trivedi, P. Dastidar, Chem Mater. 2006, 18, 3795

[35] P. Sahoo, N. N. Adarsh, G. E. Chacko, S. R. Raghavan, V. G. Puranik, P. Dastidar, Langmuir 2009, 25, 8742 .

[36] U. K. das, V. G. Puranik, P. Dastidar, Cryst. Growth. Des. 2012, Doi 10.1021/Cg301242p.

[37] J. Naskar, G. Palui, A. Banerjee, J. Phys. Chem B 2009, 113, 11787.

[38] J. Nanda, A. Banerjee, Soft Matter 2012, 8, 3380.

[39] B. Adhikari, G. Palui, A. Banerjee, Soft Matter 2009, 5, 3452.

[40] S. Prasanthkumar, A. Saeki, S. Seki, A. Ajayaghosh, J. Am. Chem. Soc. 2010, 132, 8866.

[41] S. Prasanthkumar, A. Gopal, A. Ajayaghosh, $J$. Am. Chem. Soc. 2010, 132, 13206.

[42] N. S. Saleesh Kumar, S. Varghese, G. Narayan, S. Das, Angew. Chem. Int. Ed. 2006, 45, 6317.

[43] S. Abraham, R. K. Vijayaraghavan, S. Das, Langmuir 2009, 25, 8507.

[44] S. Varghese, N. S. Saleesh Kumar, A. Krishna, D. S. Shankar Rao, S. K. Prasad, S. Das, $A d v$. Fun. Mater. 2009, 19, 2064.

[45] D. D. Prabhu, N. S. Saleesh Kumar, A. P. Sivadas, S. Varghese, S. Das, J. Phys. Chem. B 2012, 112, 13071.

[46] S. Srinivasan, S. S. Babu, V. K. Praveen, A. Ajayaghosh, Angew. Chem. Int. Ed. 2008, 47, 5746.

[47] S. Srinivasan, V. K. Praveen, R. Philip, A Ajayaghosh, Angew. Chem. Int. Ed. 2008, 47, 5750.

[48] a) A. Pal, B. S. Chhikara, A. Govindaraj, S. Bhattacharya, C. N. R. Rao, J. Mater. Chem. 2008, 18, 2593; b) S. K. Samanta, A. Pal, S. Bhattacharya, C. N. R. Rao, J. Mater. Chem. 2010, 20, 6881; c) S. K. Samanta, K. S Subrahmanyam, S. Bhattacharya, C. N. R. Rao, Chem.-Eur. J. 2012, 18, 2890.

[49] J. van Herrikhuyzen, S. J. George, M. R. J. Vos, N. A. J. M. Sommerdijk, A. Ajayaghosh, S. C. J Meskers, A. P. H. J. Schenning, Angew. Chem. Int. Ed. 2007, 46, 1825.

[50] V. R. R. Kumar, V. Sajini, T. S. Sreeprasad, V. K. Praveen, A. Ajayaghosh, T. Pradeep, Chem. Asian J. 2009, 4, 840.

[51] a) S. Bhattacharya, A. Srivastava, A. Pal, Angew. Chem. Int. Ed. 2006, 45, 2934; b) A. Pal, A. Srivastava, S. Bhattacharya, Chem.-Eur J. 2009, 15, 9169

[52] S. Datta, S. Bhattacharya, Chem. Commun. 2012, 48, 877 .
[53] a) G. Gundiah, S. Mukhopadhyay, U. G. Tumkurkar, A. Govindaraj, U. Maitra, C. N. R. Rao, J. Mater. Chem. 2003, 13, 2118; b) K. P. Kalyanikutty, M. Nikhila, U. Maitra, C. N. R. Rao, Chem. Phys. Lett. 2006, 432, 190.

[54] a) N. M. Sangeetha, S. Bhat, G. Raffy, C. Belin, A. Loppinet-Serani, C. Aymonier, P. Terech, U. Maitra, J. P. Desvergne, A. Del Guerzo, Chem. Mater. 2009, 21, 3424; b) S. Banerjee, V. M. Vidya, A. J. Savyasachi, U. Maitra, J. Mater. Chem. 2011, 21, 14693.

[55] R. K. Das, S. Bhat, S. Banerjee, C. Aymonier, A. Loppinet-Serani, P. Terech, U. Maitra, G. Raffy, J. P. Desvergne, A. Del Guerzo, J. Mater. Chem. 2011, 21, 2740.

[56] S. Bhat, U. Maitra, Chem. Mater. 2006, 18, 4224.

[57] S. Ray, A. K. Das, A. Banerjee, Chem. Commun. 2006, 2816

[58] a) B. Adhikari, A. Banerjee, Chem.-Eur. J. 2010, 16, 13698; b) B. Adhikari, J. Nanda, A. Banerjee, Soft Matter 2011, 7, 8913 .

[59] G. Palui, J. Nanda, S. Ray, A. Banerjee, Chem. -Eur. J. 2009, 15, 6902.

[60] J. Nanda, B. Adhikari, S. Basak, A. Banerjee, J. Phys. Chem. B 2012, 116, 12235.

[61] S. Roy, A. Banerjee, Rsc Adv. 2012, 2, 2105.

[62] a) B. Adhikari, A. Banerjee, Soft Matter 2011, 7, 9259; b) B. Adhikari, J. Nanda, A. Banderjee, Chem.-Eur. J. 2011, 17, 11488.

[63] S. Basak, J. Nanda, A. Banerjee, J. Mater. Chem. 2012, 22, 11658 .

[64] N. N. Adarsh, P. Sahoo, P. Dastidar, Cryst. Growth. Des. 2010, 10, 4976.

[65] N. N. Adarsh, P. Dastidar, Cryst. Growth. Des. 2011, 11, 328.

[66] M. Paul, N. N. Adarsh, P. Dastidar, Cryst. Growth. Des. 2012, 12, 4135.

[67] S. Banerjee, N. N. Adarsh, P. Dastidar, Soft Matter 2012, 8, 7623.

[68] U. K. Das, P. Dastidar, Chem.-Eur. J. 2012, 18, 13079.

[69] S. Samai, K. Biradha, Chem. Mater. 2012, 24, 1165 .

[70] A. Mallick, E. M. Schon, T. Panda, K. Sreenivas, D. D. Diaz, R. Banerjee, J. Mater. Chem. 2012, 22, 14951.

[71] S. Srinivasan, P. A. Babu, S. Mahesh, A. Ajayaghosh, J. Am. Chem. Soc. 2009, 131, 15122.

[72] Y. Hirai, S. S. Babu, V. K. Praveen, T. Yasuda, A. Ajayaghosh, T. Kato, Adv. Mater. 2009, 21, 4029.

[73] A. Das, S. Ghosh, Chem.-Eur. J. 2010, 16, 13622.

[74] M. R. Molla, A. Das, S. Ghosh, Chem.-Eur. J. 2010, 16, 10084

[75] a) A. Ajayaghosh, V. K. Praveen, C. Vijayakumar, Chem. Soc. Rev. 2008, 37, 109; b) S. S. Babu, K. K. Kartha, A. Ajayaghosh, J. Phys. Chem. Lett. 2010, 1, 3413; c) C. Vijayakumar, V. K. Praveen, K. K. Kartha, A. Ajayaghosh, Phys. Chem. Chem. Phys. 2011, 13, 4942.

[76] A. Ajayaghosh, S. J. George, V. K. Praveen, Angew. Chem. Int. Ed. 2003, 42, 332.

[77] V. K. Praveen, S. J. George, R. Varghese, C. Vijayakumar, A. Ajayaghosh, J. Am. Chem. Soc. 2006, 128, 7542 .

[78] C. Vijayakumar, V. K. Praveen, A. Ajayaghosh, Adv. Mater. 2009, 21, 2059.

[79] A. Ajayaghosh, C. Vijayakumar, V. K. Praveen, S. S. Babu, R. Varghese, J. Am. Chem. Soc. 2006, 128, 7174.

[80] A. Ajayaghosh, V. K. Praveen, C. Vijayakumar, S. J. George, Angew. Chem. Int. Ed. 2007, 46, 6260 .
[81] (a) S. Bhowmik, S. Banerjee, U. Maitra, Chem. Commun. 2010, 46, 8642; b) S. Banerjee, R. Kandanelli, S. Bhowmik, U. Maitra, Soft Matter 2011, 7, 8207

[82] (a) S. Nath, U. Maitra, Org. Lett. 2006, 8, 3239 ; b) S. Bhowmika, U. Maitra, Chem. Commun. 2012, 48, 4624

[83] S. K. Samanta, S. Bhattacharya, J. Mater. Chem. 2012, 22, 25277.

[84] S. K. Samanta, S. Bhattacharya, Chem.-Eur. J. 2012, 18, 15875 .

[85] K. V. Rao, K. K. R. Datta, M. Eswaramoorthy, S. J. George, Angew. Chem. Int. Ed. 2011, 50 , 1179

[86] K. V. Rao, K. K. R. Datta, M. Eswaramoorthy, S. J. George, Chem.-Eur. J. 2012, 18, 2184.

[87] a) D. Dasgupta, S. Srinivasan, A. Ajayaghosh, J. M. Guenet, Macromol. Symp. 2011, 303, b) D. Dasgupta, S. Srinivasan, C. Rochas, A. Thierry, A. Schroder, A. Ajayaghosh, J. M. Guenet, Soft Matter 2011, 7, 2797; c) D. Dasgupta, S. Srinivasan, C. Rochas, A Ajayaghosh, J. M. Guenet, Soft Matter 2011 , 7, 9311; d) A. T. D. Dasgupta, C. Rochas, A. Ajayaghosh, J. M. Guenet, Soft Matter 2012 8,8714 .

[88] S. Manchineella, T. Govindaraju, Rsc Adv. 2012, 10.1039/c0xx00000x.

[89] P. Koley, A. Pramanik, Soft Matter 2012, 8, 5364.

[90] A. Gopal, R. Varghese, A. Ajayaghosh, Chem. Asian J. 2012, 7, 2061.

[91] K. K. Kartha, S. S. Babu, S. Srinivasan, A. Ajayaghosh, J. Am. Chem. Soc. 2012, 134, 4834

[92] V. Bhalla, H. Singh, M. Kumar, S. K. Prasad, Langmuir 2011, 27, 15275.

[93] a) A. Vidyasagar, K. Handore, K. M. Sureshan, Angew. Chem. Int. Ed. 2011, 50, 8021; b) A. Prathap, K. M. Sureshan, Chem. Commun. 2012, 48, 5250 .

[94] M. R. Molla, S. Ghosh, Chem. Mater. 2011, 23, 95 .

[95] M. R. Molla, A. Das, S. Ghosh, Chem. Commun. 2011, 47, 8934.

[96] A. Das, M. R. Molla, A. Banerjee, A. Paul, S. Ghosh, Chem.-Eur. J. 2011, 17, 6061.

[97] M. R. Molla, S. Ghosh, Chem.-Eur. J. 2012, 18,9860 .

[98] A. Das, S. Ghosh, Chem. Commun. 2011, 47, 8922

[99] A. Das, M. R. Molla, S. Ghosh, J. Chem. Sci. 2011, 123, 963 .

[100] H. Kar, M. R. Molla, S. Ghosh, Chem Commun. 2012, 10.1039/C2CC36536G.

[101] P. Rajamalli, E. Prasad, New J. Chem. 2011, 35,1541

[102] P. Rajamalli, E. Prasad, Org. Lett. 2011, 13, 3714

[103] P. Rajamalli, E. Prasad, Soft Matter 2012, 8 8896.

[104] S. Mahesh, A. Gopal, R. Thirumalai, A Ajayaghosh, J. Am. Chem. Soc. 2012, 134, 7227

[105] R. Rajaganesh, A. Gopal, T. M. Das, A. Ajayaghosh, Org. Lett. 2012, 14, 748

[106] A. Gopal, M. Hifsudheen, S. Furumi, M. Takeuchi, A. Ajayaghosh, Angew. Chem. Int. Ed. 2012, 51, 10505 .

[107] K. V. Rao, K. Jayaramulu, T. K. Maji, S. J. George, Angew. Chem. Int. Ed. 2010, 49, 4218.

[108] K. V. Rao, S. J. George, Chem.-Eur. J. 2012, $18,14286$.

[109] A. A. Sagade, K. V. Rao, U. Mogera, S. J. George, A. Datta, G. U. Kulkarni, Adv. Mater. 2012, 25, 559. 